\title{
A simulation comparison of several procedures for testing the Poisson assumption
}

\author{
Dimitris Karlis and Evdokia Xekalaki \\ Athens University of Economics and Business, Greece
}

[Received May 1999. Revised March 2000]

\begin{abstract}
Summary. The importance of the Poisson distribution among the discrete distributions has led to the development of several hypothesis tests, for testing whether data come from a Poisson distribution against a variety of alternative distributions. An extended simulation comparison is presented concerning the power of such tests. To overcome biases caused by the use of asymptotic results for the null distribution of several tests, an extended simulation was performed for calculating the required critical points for all the tests. The results can be useful to researchers as a guide to selecting the appropriate test from several alternatives that are available.
\end{abstract}

Keywords: Bootstrap tests; Goodness of fit; Index of dispersion; Negative binomial distribution; Overdispersion; Power comparison

\section{Introduction}

The Poisson distribution plays a prominent role in the analysis of discrete data. It is widely accepted that if the data come from a pure chance mechanism then the Poisson distribution is a plausible underlying model for this mechanism and, in general, a good fit of it can be regarded as verifying the assumption that pure chance governs the situation under consideration. A good fit of the Poisson distribution also provides strong evidence of the homogeneity assumption of the population under investigation. In this respect it is very important to be able to test the Poisson hypothesis, namely to test the hypothesis $H_{0}$ : the data come from a Poisson distribution, against a general alternative hypothesis $H_{1}$ : the data do not come from a Poisson distribution.

Various test criteria have been developed and applied to test this hypothesis against various specific alternative hypotheses. The tests can be grouped into three main categories. The first contains standard goodness-of-fit tests, applied to general multinomial models. The second category contains tests derived via characteristic properties of the Poisson distribution and it can be divided into two subcategories: one based on the variance-to-mean ratio and one based on properties of the probability-generating function (PGF). The latter contains tests which are based on the difference between the models in the null and the alternative hypotheses. In what follows, we review some of them, comparing their behaviour and examining in more detail their performance in practice.

Resampling-based methodology was adopted to overcome problems caused by the inadequacy of the asymptotic results. The distribution of the test statistic under the null hypothesis is, for the majority of the tests, unknown and only asymptotic results have been derived. So, to improve the

Address for correspondence: Evdokia Xekalaki, Department of Statistics, Athens University of Economics and Business, 76 Patision Street, 10434 Athens, Greece.

E-mail: exek@aueb.gr 
comparability of the tests a preliminary extended simulation was made to find the critical values for a given significance level. These values were subsequently used for power comparison purposes.

The remainder of the paper is as follows. In Section 2 several tests proposed for testing the Poisson assumption against several alternatives are described. Section 3 describes briefly the methodology used for the power comparison, whereas in Section 4 the simulation results are reported. Concluding remarks can be found in Section 5.

\section{Tests for testing the Poisson assumption}

\subsection{Goodness-of-fit tests}

Goodness-of-fit tests contain tests that are applicable to general goodness-of-fit situations. The best-known member is the $\chi^{2}$-test which uses the statistic

$$
\chi^{2}=\sum_{x=0}^{k} \frac{\left(O_{x}-E_{x}\right)^{2}}{E_{x}}
$$

where $O_{x}$ and $E_{x}$ are the observed and expected frequencies of the value $x$. The expected values are calculated from a Poisson distribution with mean equal to the sample mean (the maximum likelihood (ML) estimate of the Poisson parameter). For calculating the test statistic we need to group cells with small expected values. The $\chi^{2}$-test has the following serious disadvantages.

(a) Its distribution is asymptotically known to be a $\chi^{2}$-distribution with $m-2$ degrees of freedom (note that we must estimate one parameter, the mean of the Poisson distribution). Unfortunately, this asymptotic result holds for large sample sizes and it depends on the grouping made. Another interesting point is that the standard result of a $\chi^{2}$-approximation holds only if the minimum $\chi^{2}$-estimate of the parameter is used (see Albrecht (1980)).

(b) The asymptotic result holds if the expected frequencies are greater than 5 (Cochran, 1954). Hence, this grouping may lead to a loss of information about the data, especially at the tails of the distributions where usually low frequencies occur. As a consequence, the test can detect only very large deviations from the Poisson distribution.

(c) The alternative hypothesis is very general with the result that if the null hypothesis is rejected very little can be said about the situation under consideration. This is the case in the majority of goodness-of-fit tests.

The main advantages of this test are that it can be easily computed and that it can be considered as nonparametric in that it can be used for a variety of distributions, allowing direct comparisons for the goodness of fit of different distributions using the value of the test statistic and the associated degrees of freedom.

Simulation studies of the distribution of the test statistic have shown that the distribution is not exactly $\chi^{2}$ and often the number of degrees of freedom differs from the actual number. To overcome this, Pahl (1969) suggested a correction of the moments of the test statistic to match the moments of the $\chi^{2}$-distribution with the appropriate degrees of freedom. Simulation-based reconstructions of the null distribution can alternatively be adopted. Hinz and Gurland (1970) and Bhalerao et al. (1980) showed that the ML estimates are inappropriate for applying the $\chi^{2}$-test.

A natural competitor of the $\chi^{2}$-test is the likelihood ratio test (LRT). To distinguish it from a test proposed in a later section, we refer to it as the multinomial LRT to emphasize that the alternative distribution is the observed multinomial distribution. The test statistic of this test also has an asymptotic $\chi^{2}$-distribution. Since no grouping is needed it is more sensitive at the tails of the distribution. An exact multinomial test is described in Horn (1977). In practice, for moderate 
sample sizes, the calculations that are needed are prohibitive. Recently, Baglivo et al. (1992) proposed an appealing method for calculating the $p$-value of a multinomial test.

To overcome problems involving low expected frequencies, Nass (1959) developed a test based on the statistic

$$
N=\frac{\sum_{i=0}^{m} \frac{O_{i}^{2}}{E_{i}}-n-(m-1)}{\sqrt{\left[\frac{m-1}{m}\left\{2 m-\frac{(m+1)^{2}+2 m}{n}+\sum_{i=0}^{m} \frac{1}{E_{i}}\right\}\right]}},
$$

where $n$ is the sample size and $m$ is the largest observed frequency. No grouping is needed. This statistic asymptotically follows the standard normal distribution. A disadvantage is that the increase in the variance makes the test rather conservative since large deviations are regarded as resulting from high variability of the data. The normal approximation was found by the simulation experiment to be poor.

Recently, some other nonparametric tests have been developed for goodness-of-fit testing. They can be considered as generalizations of the $\chi^{2}$-test in that the $\chi^{2}$-test is a special case of them. Such an important family is the family of tests that use the power divergent test statistics proposed by Read and Cressie (1988). The test statistic is defined by

$$
I^{\lambda}=\frac{1}{\lambda(\lambda+1)} \sum_{i=0}^{m} E_{i}\left\{\left(\frac{O_{i}}{E_{i}}\right)^{\lambda+1}-1\right\}, \quad \lambda \in \mathscr{R} .
$$

For all values of $\lambda, I^{\lambda}$ follows asymptotically a $\chi^{2}$-distribution with degrees of freedom equal to the number of cells minus 1 . For $\lambda=1$, equation (3) reduces to the $\chi^{2}$-test statistic whereas, for $\lambda=0$, it reduces to the multinomial LRT statistic. Several other goodness-of-fit tests are special members of this family, like the Neyman $\chi^{2}$-test $(\lambda=-2)$ and the Freeman-Tukey test $\left(\lambda=-\frac{1}{2}\right)$ among others. Read and Cressie (1988) showed that a choice of $\lambda=\frac{2}{3}$ is a very good compromise. Related to this family of tests is the family of minimum disparity goodness-of-fit tests that was proposed by Basu and Sarkar (1994).

\subsection{The variance test (or the index of dispersion test) and related tests}

Apart from the $\chi^{2}$-test, the variance test (VT) or the index of dispersion test is very common in testing the Poisson assumption. The test statistic is given by

$$
\mathrm{VT}=\sum_{i=1}^{n} \frac{\left(X_{i}-\bar{X}\right)^{2}}{\bar{X}}=(n-1) \frac{S^{2}}{\bar{X}} .
$$

This quantity is directly related to the variance-to-mean ratio, which is often referred to as the index of dispersion, multiplied by $n-1$, where $n$ is the sample size. As is well known, in the case of the Poisson distribution, the variance is equal to the mean. In the case of the binomial distribution, the index of dispersion is less than 1, and this situation is often termed underdispersion. The opposite is true for all mixed Poisson distributions whose index of dispersion is greater than 1 and shows overdispersion of the data.

A series of papers have dealt with the derivation of the expected value of the sample index of dispersion when sampling from the Poisson distribution. Bartko et al. (1968) showed that the expectation is truly equal to 1. Later Dahiya and Gurland (1969) generalized the results of Bartko et al. (1968), whereas Kharshikar (1970) derived the expected value of the index of dispersion for several members of the power series family of distributions. 
Gart and Pettigrew (1970) derived test statistics based on the property that the cumulants of the Poisson distribution are all equal to the Poisson parameter $\lambda$. Using the sample cumulants and the minimum unbiased estimator for $\lambda$, i.e. the sample mean, they derived several tests based on cumulants of different order $k$. For $k=2$ the sample cumulant reduces to the sample variance and hence a statistic that is similar to the index of dispersion is obtained. Specifically, Gart and Pettigrew (1970) proposed the use of test statistics of the form

$$
Z_{j}=\frac{k_{j}-\bar{X}}{\sqrt{ } \operatorname{var}\left(k_{j} \mid X\right)} \quad \text { for } j=2,3,4
$$

where $k_{j}$ is the $j$ th sample cumulant and $\operatorname{var}\left(k_{j} \mid X\right)$ is the variance of the $j$ th cumulant given the sum of all the observations. It is interesting that for $j=2$ the test statistic reduces to

$$
Z_{2}=\frac{S^{2}-\bar{X}}{\sqrt{ }\{2 n \bar{X}(n \bar{X}-1)\}} n \sqrt{ }(n-1) .
$$

Similarly, the tests using the third and the fourth cumulant respectively are

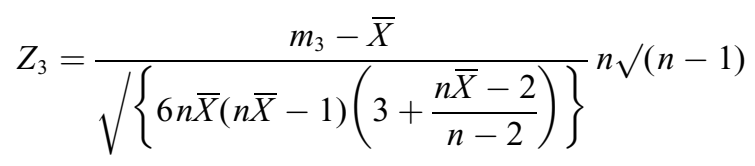

and

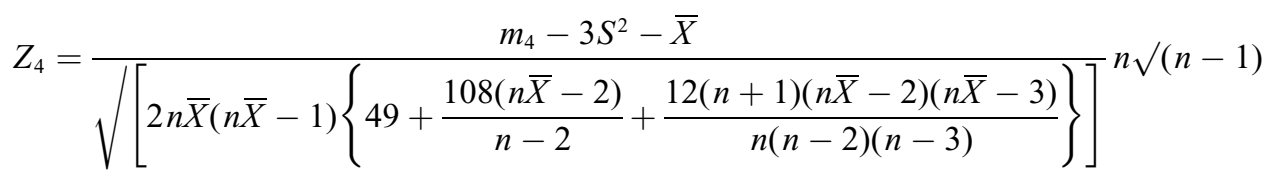

where

$$
m_{k}=n^{-1} \sum_{i=1}^{n}\left(X_{i}-\bar{X}\right)^{k}
$$

The asymptotic distribution of the test statistics of all the tests based on the sample cumulants is the standard normal distribution. Because of the increasing sampling variability of the cumulants of greater order, the use of low order cumulants is advocated.

It is known that the asymptotic distribution of the VT statistic is the $\chi^{2}$-distribution with $n-1$ degrees of freedom where $n$ is the sample size. Selby (1965) reviewed this test and pointed out that, calculating the four moments, the $\chi^{2}$-approximation is highly satisfactory for $n$ as small as 5 , as long as the mean of the Poisson distribution is greater than 5. Furthermore, when the mean is less than 5 the approximation remains fairly accurate, but this sample size is not realistic. So, the result is only asymptotically valid and it does not work satisfactorily in practice.

Anderson and Siddiqui (1994) derived the first four moments of the index of dispersion for some discrete distributions. They also tried to develop the sampling distribution. Instead of using the bad $\chi^{2}$-approximation, they proposed to use a general two-parameter gamma distribution, matching the first two moments of the index of dispersion with the moments of the gamma distribution. They also considered other approximations and conducted a simulation experiment to base their proposals on. For the Poisson distribution, the sampling distribution of the variance-tomean ratio (which is the VT statistic divided by $n-1$ ) can be well represented by a $\chi^{2}$-distribution with 1 degree of freedom. However, the VT, which is a multiple of the index of dispersion, cannot be well approximated by a $\chi^{2}$-distribution with $n-1$ degrees of freedom. It would, therefore, be 
reasonable to consider the use of the index of dispersion as the test statistic. The advantages of this test are that it can be used with small samples, it is more sensitive than the usual $\chi^{2}$-test and using the value of its test statistic we can detect the sort of deviation from the Poisson assumption (e.g. overdispersion or underdispersion). In practice, the VT can lead to results that are more reliable than those provided by a $\chi^{2}$-test (see the discussion in Cochran (1954)).

The power of the VT depends on the distribution under the alternative hypothesis. Bateman (1950) treated the case where the distribution under the alternative hypothesis is Neyman's contagion distribution. Darwin (1957) treated various other choices of alternative distributions such as the Neyman distribution, the double-Poisson distribution and the negative binomial distribution. Selby (1965) calculated the moment-generating function of the index under the general hypothesis of a mixed Poisson distribution. This corresponds to a non-central $\chi^{2}$-distribution with the noncentrality parameter depending on the specific distribution. Various ramifications of this test are given in Potthoff and Whittinghill (1966) and Collings and Margolin (1985). They both used this test in more complicated situations. In Potthoff and Whittinghill (1966) a review of the $\chi^{2}$-test was made as well as a comparison of the two tests. Kim and Park (1992) extended the work of Collings and Margolin (1985) to derive locally optimal tests for the Poisson distribution against the negative binomial distribution for several other situations. Perry and Mead (1979) examined the power of the variance test when it is used to detect a spatial pattern.

If the assumption of a Poisson distribution holds then the variance of the population should be equal to the mean. Hence the null hypothesis can be rewritten as $H_{0}: \sigma^{2}=\mu$. To test this hypothesis the mean and the variance of the statistic $S^{2}-\bar{X}$ must be determined, to consider the standard normal distribution approximation to its distribution. Potthoff and Whittinghill (1966) and Böhning (1994) showed that the test statistic is

$$
O_{2}=\sqrt{ }\left(\frac{n-1}{2}\right)\left(\frac{S^{2}}{\bar{X}}-1\right)=\frac{\sum_{i}\left(X_{i}-\bar{X}\right)^{2}}{\bar{X} \sqrt{ }\{2(n-1)\}}-\frac{\sqrt{ }(n-1)}{\sqrt{ } 2} .
$$

This test is, among all locally unbiased tests for testing the Poisson assumption against a mixed Poisson distribution, asymptotically locally most powerful with respect to a gamma mixing distribution, i.e. against a negative binomial distribution (Potthoff and Whittinghill, 1966). Bartoo and Puri (1967) examined this optimality and showed that a further restriction must be imposed on the mixing distribution. Moran (1973) extended the results of Bartoo and Puri (1967), examining the case when the mixing distribution is symmetric (zero third central moment).

Zelterman (1988) derived a test for testing the null hypothesis of a one-parameter distribution against an alternative hypothesis of a mixture on this parameter with the same mean (central mixture). For the Poisson case, the test is testing the Poisson hypothesis against a mixed Poisson hypothesis. The test statistic given by

$$
Z=\frac{\sum_{i}\left(X_{i}-\bar{X}\right)^{2}}{\bar{X} \sqrt{ }(2 n)}-\sqrt{\left(\frac{n}{2}\right)}
$$

follows asymptotically a standard normal distribution. It is very interesting that the test statistics given in equations (5), (8) and (9) are quite similar and only small differences are expected to exist in real applications. A variant of the VT is also discussed in Kyriakoussis et al. (1998).

Rayner and McIntyre (1985) derived the score test for testing the Poisson distribution assumption against the alternative assumption of the generalized Poisson distribution. The generalized Poisson distribution has probability function 


$$
P(X)=\lambda(\lambda+x \theta)^{x-1} \exp \{-(\lambda+x \theta)\} / x !
$$

for $x=0,1, \ldots, \lambda>0$ and $|\theta|<1$ and $P(x)=0$ if $x \geqslant m$ and $\lambda+\theta m \leqslant 0$.

This distribution reduces to the Poisson distribution for $\theta=0$ and it has been examined in detail by Consul (1989). It is interesting that the variance-to-mean ratio of this distribution may take values that are less or greater than 1 and thus it may represent underdispersed or overdispersed data. The test statistic proposed for testing the Poisson assumption against a generalized Poisson distribution alternative is

$$
W=\left(\frac{S^{2}}{\bar{X}}-1\right)^{2} \frac{n}{2}
$$

which approximately follows a $\chi^{2}$-distribution with 1 degree of freedom. This approximation is sufficient for large sample sizes and large values of the mean. Note the great similarity to the $\mathrm{O}_{2}$ test statistic given in equation (8). The $\mathrm{O}_{2}$ test statistic is the square root of the $W$-statistic if $n$ is replaced by $n-1$.

Summarizing we can say that the VT test is the locally most powerful unbiased test for a negative binomial alternative hypothesis, the $Z$-test for a general central mixture alternative hypothesis and the $W$-test for the generalized Poisson distribution alternative hypothesis. Another test for such hypothesis testing is discussed in Henze and Klar (1995).

Henze and Klar (1996) showed that the VT cannot be used as a diagnostic when it rejects the null hypothesis. When we reject the null hypothesis of a Poisson distribution, the test statistic cannot indicate the form of the true, but unknown, underlying distribution (overdispersed, underdispersed or equidispersed). They proposed a rescaled alternative statistic, which is given by

$$
S^{*}=\frac{\bar{X}(\mathrm{VT}-n)^{2}}{\sum_{i=1}^{n}\left\{\left(X_{i}-\bar{X}\right)^{2}-X_{i}\right\}} .
$$

Smooth tests have been proposed by Rayner and Best (1988). They proposed the use of Poisson-Charlier polynomials of order $k$ for testing the Poisson assumption. Depending on the choice of $k$ for the polynomials used, a broad class of smooth tests can be constructed. The test statistic is defined as

$$
S_{k}=\sum_{i=2}^{k} V_{i}^{2}
$$

where $V_{i}(i=2, \ldots, k)$ are defined via the Poisson-Charlier polynomials by

$$
V_{i}=\frac{1}{\sqrt{ } n} \sum_{j=1}^{n} h_{i}\left(X_{j}, b\right)
$$

where $h_{i}(x, b)$ is the $i$ th term of a Poisson-Charlier polynomial defined as

$$
h_{i}(x, b)=\sqrt{ }\left(\frac{b^{i}}{i !}\right) \sum_{u=0}^{i}(-1)^{i-u}\left(\begin{array}{c}
i \\
u
\end{array}\right) u ! \frac{1}{b^{u}}\left(\begin{array}{l}
x \\
u
\end{array}\right) .
$$

It is easily checked that $h_{0}(x, b)=1$, for any value of $x$ and $b$. Similarly, $h_{1}(x, b)=\sqrt{ } b(x / b-1)$.

A useful recurrence relationship is

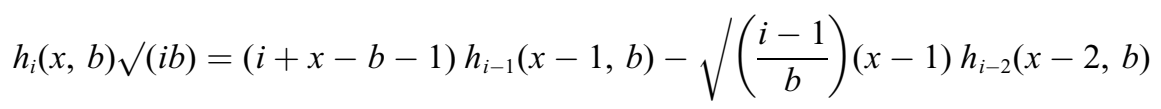


with the initial values $h_{0}(x, b)$ and $h_{1}(x, b)$ defined above for all the values of $x$. The value of $b$ is the ML estimate for the parameter of the distribution, i.e. the sample mean. For $k=2$ the test reduces to the test proposed by Zelterman and it is given in equation (9).

Rayner and Best (1988) showed that, asymptotically, the test statistic follows a $\chi^{2}$-distribution with 4 degrees of freedom. However, this approximation is not very satisfactory in practice. Recently, Best and Rayner (1999) made a comparison of the performance of test (12) with that of some other tests used in the literature.

Gupta et al. (1994) proposed another test statistic, which uses the coefficients of skewness and kurtosis. In this test the alternative hypothesis is that the data come from an infinitely divisible distribution. Gupta et al. (1994) showed that, if $\alpha$ and $b$ are the coefficients of skewness and kurtosis respectively, the statistic

$$
T=\frac{1}{2} \sqrt{\left(\frac{n}{1+24 \bar{X}+6 \bar{X}^{2}}\right)} \frac{m_{2}\left(m_{4}-3 m_{2}^{2}\right)-m_{3}}{\bar{X}^{2}}
$$

tends to a standard normal distribution.

Cox (1983) in his paper about overdispersion suggested another test, which is a generalization of the VT. This test is valid for all of the exponential family of distributions, either discrete or continuous. Gelfand and Dalal (1990) discussed the problem of overdispersion in detail and derived a test for overdispersion that was similar to Cox's. They also provided a graphical test for overdispersion based on certain properties of overdispersion models. Other graphical methods for checking the Poisson assumption can be found in Ord (1967), Lindsay (1986), Santner and Duffy (1989), Hoaglin (1980) and Lindsay and Roeder (1992). Pettigrew and Mohler (1967) proposed another test based on the range. Kim (1988) examined the situation under which the locally most powerful unbiased test exists.

\subsection{Other tests}

\subsubsection{A Kolmogorov-Smirnov type of test}

The Kolmogorov-Smirnov (KS) test is known to be a powerful goodness-of-fit test for continuous distributions. Conover (1972) described the calculation for the level of significance for the KS test for discontinuous data, leading to an exact KS test. Wood and Altavela (1978) derived the asymptotic distribution of the test statistics for large sample sizes. Campbell and Oprian (1979) treated the case of the Poisson distribution when the parameter must be estimated from the data. They reported the required critical values for the test via simulation.

Suppose that $H(x)=P(X \leqslant x)$ is the cumulative distribution function of the distribution assumed and that $S_{N}(x)$ is the empirical cumulative distribution function of the sample obtained as the proportion of observations in the sample with value less than or equal to $x$. Then, the test statistic is

$$
d=\max \left|H(x)-S_{N}(x)\right| \quad x=0,1, \ldots
$$

The distribution of the test statistic is not known in a closed form and simulation methods are required for its construction. Properties of the test are discussed in Henze (1996).

\subsubsection{Cramér-von Mises type of tests}

Henze (1996) and Spinelli and Stephens (1997) developed Cramér-von Mises statistics for testing the Poisson hypothesis. These statistics are based on squared differences between the theoretical and the empirical cumulative functions, suitably weighted. Spinelli and Stephens (1997) proposed 
and examined three different statistics, which are given by

$$
\begin{gathered}
\mathrm{CVM}_{1}=n^{-1} \sum_{j=0}^{M} Z_{j}^{2} p_{j}, \\
\mathrm{CVM}_{2}=n^{-1} \sum_{j=0}^{M} \frac{Z_{j}^{2} p_{j}}{H(j)\{1-H(j)\}}, \\
\mathrm{CVM}_{3}=n^{-1} \sum_{j=0}^{M} Z_{j}^{2}
\end{gathered}
$$

where $Z_{j}=\sum_{i=0}^{j}\left(O_{i}-E_{i}\right)$ and $p_{j}$ is the theoretical probability of the value $j$. We can see that the three tests put different weights on the squared differences; the test given by equation (18) does not use any weighting of the differences. For all the tests, the summations can be stopped at a value $M$ such that $p_{M}<0.0001$. The error committed by this truncation is negligible.

The test proposed by Henze (1996) uses the observed relative frequencies as weights instead of the theoretical frequencies used in equation (16). This test is given by

$$
\mathrm{CVM}_{4}=n^{-2} \sum_{j=0}^{M} Z_{j}^{2} O_{j}
$$

\subsubsection{Tests based on the empirical probability-generating function}

A large number of testing procedures have been developed based on test statistics that utilize properties of the PGF of the Poisson distribution.

Kocherlakota and Kocherlakota (1986) proposed a test statistic for discrete distributions based on the empirical probability-generating function (EPGF). The EPGF $\phi_{n}(t)$ is defined as

$$
\phi_{n}(t)=\frac{1}{n} \sum_{i=1}^{n} t^{X_{i}}
$$

According to the test procedure proposed, $\phi_{n}(t)$ is compared with the theoretical PGF, say $\phi(t)$. Kocherlakota and Kocherlakota (1986) proposed the use of the test statistic

$$
K=\sqrt{ } n \frac{\phi_{n}(t)-\exp \{\bar{x}(t-1)\}}{\exp \left\{\bar{x}\left(t^{2}-1\right)\right\}-\exp \{2 \bar{x}(t-1)\}\left\{1+\bar{x}(t-1)^{2}\right\}} .
$$

This test statistic asymptotically follows a standard normal distribution. This test has one disadvantage. It depends on the choice of the value of $t$. Even though the test statistic is not so sensitive to the choice of $t$, Kocherlakota and Kocherlakota (1986) showed that the test can be improved by evaluating the test statistic for more than one value of $t$, at the cost of increasing the numerical complexity. For the case of using the test statistic given above they proposed the use of a small positive value of $t$ near 0 .

To overcome the problem related to the choice of $t$, Rueda et al. (1991) and Rueda and O'Reilly (1999) proposed the use of the squared difference between the true PGF and the EPGF, integrating over the interval $(0,1)$. The test statistic is

$$
d_{n}(\lambda)=\frac{1}{n} \sum_{i=1}^{n} \sum_{j=1}^{n} \frac{1}{X_{i}+X_{j}+1}-2 \exp (-\lambda) \sum_{i=1}^{n} T\left(X_{i}, \lambda\right)+n \frac{1-\exp (-2 \lambda)}{2 \lambda}
$$

where

$$
T(x, \lambda)=\int_{0}^{1} t^{x} \exp (\lambda t) \mathrm{d} t
$$


$T(x, \lambda)$ may be calculated recursively by using

$$
T(x, \lambda)=\frac{\exp (\lambda)}{\lambda}-\frac{x}{\lambda} T(x-1, \lambda)
$$

and

$$
T(0, \lambda)=\frac{\exp (\lambda)-1}{\lambda}
$$

For applying the test procedure $\lambda$ is replaced by its ML estimate. Similar procedures were discussed in Epps (1995).

Baringhaus and Henze (1992) also proposed a test based on the EPGF. They based their test on the characteristic property of the Poisson distribution that $\partial \phi(t) / \partial t=\lambda \phi(t)$, where $\phi(t)$ is a proper PGF. Hence, a test procedure can be developed by considering $\partial \phi(t) / \partial t=\lambda \phi(t)$ and integrating over the interval $(0,1)$. The test statistic obtained is

$$
T_{n}=\frac{1}{n} \sum_{i=1}^{n} \sum_{j=1}^{n}\left(\frac{\bar{X}^{2}}{X_{i}+X_{j}+1}+\frac{X_{i} X_{j}}{X_{i}+X_{j}-1}\right)-\left(n-f_{0}\right) \bar{X},
$$

where $f_{0}$ is the observed proportion of 0 . Baringhaus and Henze (1992) constructed tables of the critical values of this test statistic via simulation. They also showed that the power of the test tends to 1 as the sample size increases for any form of alternative distribution with a finite first moment.

Nakamura and Perez-Abreu (1993) based their test on the property that the PGF $\phi(t)$ of the Poisson distribution satisfies the relationship $\partial[\log \{\phi(t)\}] / \partial t=\lambda$ and, hence, its second derivative is equal to 0 . The test statistic proposed is

$$
\mathrm{NP}_{n}=\frac{1}{n^{3}} \frac{\sum_{i, j, k, l=1}^{n} X_{i}\left(X_{i}-X_{j}-1\right) X_{k}\left(X_{k}-X_{l}-1\right) I_{\left(X_{i}+X_{j}=X_{k}+X_{l}\right)}}{\bar{X}^{1.45}}
$$

where $I_{x}$ is the indicator function. They constructed tables of the critical values of statistic (23) via simulation and showed that the limiting form of the distribution of this test statistic is independent of the value of $\lambda$.

\subsubsection{A test based on the integrated distribution function}

Recently, Klar (1999) proposed a test based on the integrated distribution function and its empirical counterpart. The integrated distribution function is defined for positive data as

$$
\Psi_{X}(t)=E(X-t)^{+}=\int_{t}^{\infty}\{1-H(x)\} \mathrm{d} x .
$$

For discrete data, as in our case, it is given by $\Psi(t)=\sum_{k=[t]+1}^{\infty}(k-t) p_{k}$ where $[t]$ denotes the integral part of $t$. The empirical counterpart is calculated from the data as

$$
\hat{\Psi}_{n}(t)=n^{-1} \sum_{i=1}^{n}\left(X_{i}-t\right) I_{\left(X_{i}>t\right)} .
$$

The test statistic proposed by Klar (1999) is

$$
\mathrm{EIDF}=\sup _{t \geqslant 0}\left\{\left|\Psi(t)-\hat{\Psi}_{n}(t)\right| \sqrt{ } n\right\},
$$

which is similar to the Kolmogorov-type statistic, when the cumulative distribution function is replaced by the integrated distribution function. The properties of the statistic are described in 
Klar (1999). The parametric bootstrap is needed for constructing the distribution of the test statistic.

\subsubsection{The likelihood ratio tests and the Hellinger deviance test}

The LRT is a common method for testing hypotheses which can be considered as nested, in the sense that the model under the null hypothesis is a special case of the model under the alternative. The LRT is widely used for finite mixtures, to test whether the simple model is adequate against the alternative hypothesis that a 2-finite mixture is more appropriate (see, for example, Titterington et al. (1985)). Using this methodology we can apply the LRT to test the Poisson assumption against a 2-finite Poisson mixture assumption. The test statistic is given by

$$
L=2\left(L_{1}-L_{0}\right)
$$

where $L_{i}, i=0,1$, are the maximized log-likelihoods for the hypotheses in $H_{i}$. Since the values of the parameters supporting the null hypothesis lie on the boundary of the parameter space the standard asymptotic result of a $\chi^{2}$-distribution is not applicable. Several researchers have described a bootstrap approach for constructing the null distribution of the test statistic (see, for example, McLachlan (1987), Böhning et al. (1994) and Karlis and Xekalaki (1999) among others).

Recently, Karlis and Xekalaki (1998a) proposed a similar test, which is based on the Hellinger distance rather than on the likelihood. The test statistic is given by

$$
\mathrm{HDT}=4 n\left(\mathrm{HD}_{0}-\mathrm{HD}_{1}\right) \text {, }
$$

where $\mathrm{HD}_{i}, i=0,1$, are the minimized Hellinger distances for the distributions under the two hypotheses.

The minimization of the Hellinger distance can be achieved by a simple iterative scheme described in Karlis and Xekalaki (1998b). It has been shown that HDT is robust against outliers.

\subsubsection{Further tests}

Lee (1998) proposed some other tests for the Poisson assumption. The tests are based on expanded classes of distributions, in which the Poisson distribution belongs, depending on the specific value of a parameter. Thus the hypothesis is tested that this parameter is 0 , leading to the Poisson distribution. These classes include the Katz family and the double-exponential family of distributions.

The former leads to a variant of the VT statistic. The latter is defined by Efron (1986), by introducing a dispersion parameter to the exponential family. It enjoys exponential family properties for both parameters (the mean and dispersion). Lee (1998) derived the test statistic for the Poisson distribution given by

$$
\mathrm{EF}=2 \sum_{i=1}^{n}\left\{\bar{X}-X_{i}+X_{i} \ln \left(\frac{X_{i}}{\bar{X}}\right)\right\}=2 \sum_{i=1}^{n} X_{i} \ln \left(\frac{X_{i}}{\bar{X}}\right),
$$

to test whether the dispersion parameter equals 0 .

\section{A power comparison of several tests}

\subsection{Description of the simulation comparison}

In what follows, the power of the test procedures presented in the preceding sections for testing the hypothesis that the data come from a Poisson distribution is examined to compare their power 
and performance. Table 1 contains all the tests that are compared. It is important to note that this seems to constitute the first attempt in the statistical literature for such a comparison between so many tests for testing the Poisson assumption.

In making a comparative evaluation of testing procedures we seek certain desirable features such as high power, applicability and ease of computation. However, we seek tests whose test statistic has a known (and simple) distribution, which is easily applicable. Unfortunately, the majority of the tests proposed do not have a standard null distribution for their test statistic, and

Table 1. Tests compared via the simulation experiment

\begin{tabular}{|c|c|c|c|c|}
\hline Name of test & Reference & Critical region & Alternative & Test statistic \\
\hline VT & Cochran (1954) & $\begin{array}{l}\text { Depends on } \\
\text { alternative }\end{array}$ & $\begin{array}{l}\text { Overdispersed or } \\
\text { underdispersed }\end{array}$ & (4) \\
\hline Böhning & Böhning (1994) & $\begin{array}{l}\text { Depends on } \\
\text { alternative }\end{array}$ & $\begin{array}{l}\text { Overdispersed or } \\
\text { underdispersed }\end{array}$ & (8) \\
\hline Zelterman & Zelterman (1988) & $\begin{array}{l}\text { Depends on } \\
\text { alternative }\end{array}$ & Mixtures & (9) \\
\hline 2nd cumulant & Gart and Pettigrew (1970) & $\begin{array}{l}\text { Depends on } \\
\text { alternative }\end{array}$ & $\begin{array}{l}\text { Overdispersed or } \\
\text { underdispersed }\end{array}$ & (5) \\
\hline 3rd cumulant & Gart and Pettigrew (1970) & Both tails & No Poisson & (6) \\
\hline 4th cumulant & Gart and Pettigrew (1970) & Both tails & No Poisson & (7) \\
\hline $\begin{array}{l}\text { Kocherlakota with } \\
t \quad=-0.05\end{array}$ & $\begin{array}{l}\text { Kocherlakota and } \\
\text { Kocherlakota (1986) }\end{array}$ & Both tails & No Poisson & (20) \\
\hline $\begin{array}{l}\text { Kocherlakota with } \\
t=0.05\end{array}$ & $\begin{array}{l}\text { Kocherlakota and } \\
\text { Kocherlakota (1986) }\end{array}$ & Both tails & No Poisson & $(20)$ \\
\hline $\begin{array}{l}\text { Kocherlakota with } \\
t=0.125\end{array}$ & $\begin{array}{l}\text { Kocherlakota and } \\
\text { Kocherlakota (1986) }\end{array}$ & Both tails & No Poisson & $(20)$ \\
\hline Nass & Nass (1959) & Both tails & No Poisson & (2) \\
\hline Gupta & Gupta et al. (1994) & Left tail & Infinite divisible distribution & (13) \\
\hline Baringhaus & $\begin{array}{l}\text { Baringhaus and Henze } \\
\text { (1992) }\end{array}$ & Right tail & No Poisson & (22) \\
\hline Nakamura & $\begin{array}{l}\text { Nakamura and Perez-Abreu } \\
\text { (1993) }\end{array}$ & Right tail & No Poisson & (23) \\
\hline Rueda & Rueda et al. (1991) & Right tail & No Poisson & $(21)$ \\
\hline KS & $\begin{array}{l}\text { Campbell and Oprian } \\
\text { (1979) }\end{array}$ & Right tail & No Poisson & (15) \\
\hline McIntyre & $\begin{array}{l}\text { Rayner and McIntyre } \\
\text { (1985) }\end{array}$ & Right tail & No Poisson & (11) \\
\hline$\chi^{2}$ & Cochran (1954) & Right tail & No Poisson & (1) \\
\hline $\begin{array}{l}\text { Power divergent family, } \\
\quad \lambda=2 / 3\end{array}$ & Read and Cressie (1988) & Right tail & No Poisson & (3) \\
\hline LRT & Titterington et al. (1985) & Right tail & Poisson mixture & $(25)$ \\
\hline Hellinger deviance test & $\begin{array}{l}\text { Karlis and Xekalaki } \\
\text { (1998a) }\end{array}$ & Right tail & Poisson mixture & (26) \\
\hline Rayner & Rayner and Best (1988) & Right tail & No Poisson & (13) \\
\hline Cramér-von Mises 1 & $\begin{array}{l}\text { Spinelli and Stephens } \\
\text { (1997) }\end{array}$ & Right tail & No Poisson & (16) \\
\hline Cramér-von Mises 2 & $\begin{array}{l}\text { Spinelli and Stephens } \\
\text { (1997) }\end{array}$ & Right tail & No Poisson & (17) \\
\hline Cramér-von Mises 3 & $\begin{array}{l}\text { Spinelli and Stephens } \\
\text { (1997) }\end{array}$ & Right tail & No Poisson & (18) \\
\hline Cramér-von Mises 4 & Henze (1996) & Right tail & No Poisson & (19) \\
\hline Rescaled VT & Henze and Klar (1996) & Right tail & $\begin{array}{l}\text { Overdispersed or } \\
\text { underdispersed }\end{array}$ & (12) \\
\hline $\begin{array}{l}\text { Empirical integrated } \\
\text { distribution function }\end{array}$ & Klar (1999) & Right tail & No Poisson & (24) \\
\hline $\begin{array}{l}\text { Efron double-exponential } \\
\text { family }\end{array}$ & Lee (1998) & Right tail & No Poisson & (27) \\
\hline
\end{tabular}


the critical values are either unknown or they have been tabulated in a limited form by some researchers.

The aim is to find the test with the highest power, i.e. the test with the smallest probability of falsely rejecting the null hypothesis. Since exact power calculations are not possible, we are restricted to the use of empirical power calculations via extensive simulation. The empirical power is defined as the proportion of times that the null hypothesis is rejected when the data were generated from the alternative hypothesis.

The simulation experiment was carried out in two steps. In the first step the critical values for all the tests and all the distributions and sample sizes involved were obtained via simulation. The method employed for deriving these critical values is that utilized by Efron and Tibshirani (1993) for the estimation of a percentile.

So, using the parametric bootstrap, $B=5000$ values of the test statistic were simulated for a given sampling distribution and sample size. The $\alpha$-percentile can be consistently estimated by the $d$ th order statistic of the distribution of the $B$-values of the test statistic, where $d=$ $\left[a^{*} B / 100\right]$, and $[a]$ denotes the integer part of $a$. This procedure was repeated 20 times and the critical values derived were the mean of these 20 repetitions.

This has also been done for tests for which the asymptotic form of the distribution of the test statistic is known, e.g. the $\chi^{2}$-test. In using this approach we aim to reduce the bias of using nonaccurate critical values for the power comparisons.

Having obtained the critical values, we may proceed with the power comparison. From each alternative, 10000 samples of given size were generated, and the test statistics were calculated. For values of the statistic in the rejection area, as defined by the critical values obtained from the first step, the null hypothesis was rejected. The reported empirical power is the proportion of times that the null hypothesis was rejected.

This approach is different from that described in Stute et al. (1993) which is easier to use in practice. However, because of the great extent of our simulation comparison, the approach described below was followed. As verified by a smaller experiment, our approach does not introduce any systematic bias into the power of the tests and clearly does not affect the comparisons between the tests.

For each null distribution, several alternatives were examined. Because the alternative hypotheses of the tests under study differed, we considered all the alternatives specified for each test to examine the consequences of their false use.

Three values were considered for the Poisson parameter, namely 1, 3 and 5, whereas the sample sizes used were 50, 100 and 500. The level of significance was set to 5\% $(\alpha=0.05) .10000$ simulations were used for deriving the empirical power for each test and combination of Poisson parameter and sample size.

The alternatives were chosen to match the first moment and to represent some patterns of deviation from the Poisson distribution. Alternatives with the variance equal to the mean were also considered. The results for the equidispersed case are reported separately.

A table look-up method was used for simulating from the distributions involved (see, for example, Devroye (1992)). All the programs were run on a Sun workstation computer. The results of this extensive simulation experiment are presented in Section 4. In the next two subsections, the alternative distributions that were used are described.

\subsubsection{Overdispersed and underdispersed alternatives}

For the case of $H_{0}$ : the data come from a Poisson $(\lambda)$ distribution, the alternatives used were

(a) a 2 -finite Poisson mixture with parameters $(0.5,0.95 \lambda, 1.05 \lambda)$ (this distribution is slightly 
different from the Poisson distribution and the index of dispersion is $1+0.0025 \lambda$, i.e. it differs very little from the Poisson distribution),

(b) a 2 -finite Poisson mixture with parameters $(0.5,0.5 \lambda, 1.5 \lambda)$ (the index of dispersion is $1+0.25 \lambda$, and for large values of $\lambda$ the distribution is bimodal and very overdispersed),

(c) a 2-finite Poisson mixture with parameters $(0.8,0.9 \lambda, 1.4 \lambda)$ (the index of dispersion is $1+0.04 \lambda$, but it is more skewed than the Poisson distribution),

(d) a negative binomial distribution with index of dispersion equal to 1.1,

(e) a Poisson-inverse Gaussian distribution with index of dispersion equal to 1.1,

(f) a generalized Poisson distribution with index of dispersion equal to 1.1,

(g) a negative binomial distribution with index of dispersion equal to 1.5 ,

(h) a Poisson-inverse Gaussian distribution with index of dispersion equal to 1.5,

(i) a generalized Poisson distribution with index of dispersion equal to 1.5 ,

(j) a binomial distribution with $N=3 \lambda+5$ and a value of $p$ such that $N p$ matches the first moment (through the use of the binomial distribution we have an idea of the behaviour of the tests in the case of underdispersion),

(k) a generalized Poisson distribution with index of dispersion equal to 0.9 ,

(l) a 2 -finite Poisson mixture with parameters $(0.37,0.6 \lambda, 1.2 \lambda)$ (the index of dispersion is $1+0.04 \lambda$, but it is more skew than the Poisson distribution),

(m) a negative binomial distribution with index of dispersion equal to $1+0.25 \lambda$ and

(n) a Poisson-inverse Gaussian distribution with index of dispersion equal to $1+0.25 \lambda$.

Overdispersed distributions (d)-(f) with slight overdispersion were chosen to represent some different patterns. For example, the Sichel distribution is known to have a longer right-hand tail than the negative binomial distribution has (Sichel, 1975). The value of the overdispersion parameter considered for distributions (g)-(i) is larger, to allow an examination of cases where we have a greater departure from the Poisson distribution.

The 14 forms of alternative distributions considered refer to both underdispersed and overdispersed situations.

Table 2 contains some descriptive measures for some of the distributions considered in the simulations. These can be used to obtain the values of the parameters for the distributions in the alternative hypothesis.

\subsubsection{Alternatives with variance equal to the mean}

Henze and Klar (1996) showed that the VT and its variants do not perform well when the actual distribution of the data has the variance equal to the mean. To examine all the tests in this case, also, we considered some alternatives that satisfy this property. The beta-binomial distribution, with suitably chosen parameters, was used.

The beta-binomial distribution arises as a mixture on the parameter $p$ of the binomial distribution (see for example Johnson et al. (1992)). In Table 2 we can see that, depending on the different values of the parameter $N$ of the beta-binomial distribution, we can find combinations of values for the remaining parameters satisfying the required property. For a fixed value of the mean, we consider three values for $N$. As the value of $N$ increases, the resulting beta-binomial distribution tends to be indistinguishable from the Poisson distribution with the same mean. The smaller the value of $N$ the larger the difference from the Poisson distribution is. So, the three chosen values correspond to beta-binomial alternative distributions with varying 'difference' from the Poisson distribution.

In the case of a discrete uniform distribution as an alternative, even when the variance equals the mean, the difference from the Poisson distribution will be rather great, and hence the sensitivity of the test cannot be examined in detail. 
Table 2. Distributions used in the simulation, their means, variances and indices of dispersion $\dagger$

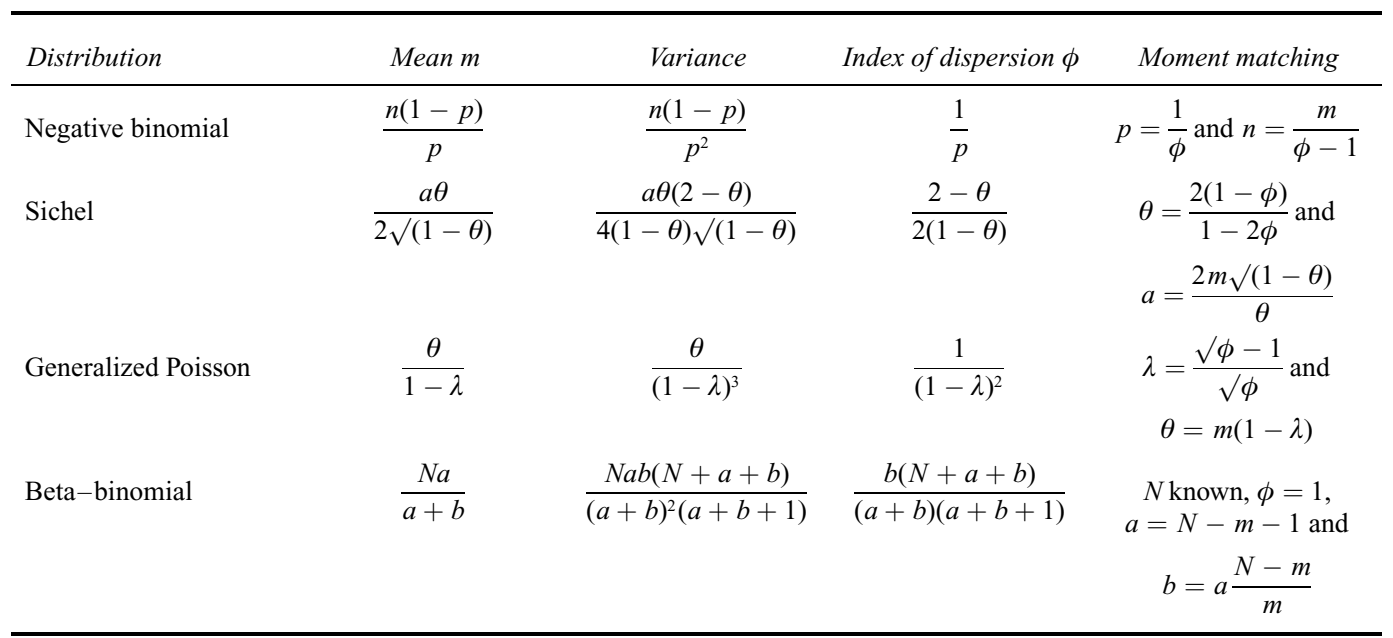

†The last column contains the values of the parameters that lead to the given values of the mean $m$ and the index of dispersion $\phi$.

The combinations of parameters $(N, \alpha, b)$ of the beta-binomial distribution considered for each value of the Poisson parameter $\lambda$ were, for $\lambda=1,(6,4,20),(4,2,6)$ and $(3,1,2)$, for $\lambda=3$, $(12,8,24),(8,4,6.67)$ and $(5,1,0.67)$, and, for $\lambda=5,(18,12,31.2),(12,6,8.4)$ and $(7,1,0.4)$.

\section{The results of the simulation study}

\subsection{Overdispersed and underdispersed alternatives}

Let us now examine the results reported in Tables 3-11. A first glance reveals that the test suggested by Gupta is inferior in all cases and its power is very low. Similar is the behaviour of the fourth-cumulant test, which is almost always inferior to the other tests. An explanation for this might be the high variability of the fourth sample cumulant which the test uses.

The performances of all the variants of the VT are very similar. This was expected because all these tests are highly correlated. Differences between them are mainly due to sampling errors.

We start from the simplest case, the underdispersed alternatives (j) and (k). As expected, the LRT and the Hellinger deviance test have zero power since they test the Poisson model against the 2-finite Poisson model, i.e. against the overdispersion hypothesis. Note that the index of dispersion is 0.9 , which is not very far from 1 . The VT performs better. It is interesting that the tests based on the PGF perform better when the index of dispersion decreases, as is the case where $\lambda=5$. A careful examination of the results reveals that the VT and its variants exhibit a better performance.

The case where the alternative distributions show overdispersion is more difficult. As expected, the power increases as the index of dispersion increases. For large values of it (e.g. 1.25) and large sample sizes, all the methods perform satisfactorily. In contrast, low values of the index of dispersion, as in the case implied by alternative 1, result in a decrease in the power of the tests even with large sample sizes. A close examination of the tables reveals that two groups of tests perform well: the VT test and its variants and the LRT and Hellinger deviance test.

Another interesting comparison is between the $\chi^{2}$-test and the power divergent test with $\lambda=\frac{2}{3}$. The results show that their performances are quite similar, and any differences can be attributed to sampling errors. So, choosing one from them is not important. The test of Nass, which is a variant 
Table 3. Calculated power for all the tests employed in the simulation study and for all the alternative distributions used $\dagger$

\begin{tabular}{|c|c|c|c|c|c|c|c|c|c|c|c|c|c|c|}
\hline \multirow[t]{2}{*}{ Test } & \multicolumn{14}{|c|}{ Powers for the following alternative distributions: } \\
\hline & (a) & (b) & (c) & (d) & (e) & $(f)$ & (g) & (h) & (i) & $(j)$ & ( $k$ ) & (l) & (m) & (n) \\
\hline 2nd cumulant & 0.049 & 0.310 & 0.078 & 0.122 & 0.132 & 0.148 & 0.606 & 0.599 & 0.570 & 0.157 & 0.125 & 0.112 & 0.296 & 0.308 \\
\hline VT & 0.054 & 0.339 & 0.086 & 0.130 & 0.149 & 0.165 & 0.620 & 0.621 & 0.590 & 0.145 & 0.110 & 0.123 & 0.315 & 0.323 \\
\hline Böhning & 0.060 & 0.366 & 0.093 & 0.139 & 0.158 & 0.176 & 0.640 & 0.641 & 0.607 & 0.142 & 0.109 & 0.133 & 0.327 & 0.338 \\
\hline Zelterman & 0.049 & 0.309 & 0.077 & 0.121 & 0.130 & 0.148 & 0.599 & 0.596 & 0.570 & 0.157 & 0.125 & 0.111 & 0.294 & 0.306 \\
\hline 3rd cumulant & 0.054 & 0.136 & 0.054 & 0.070 & 0.085 & 0.098 & 0.390 & 0.413 & 0.414 & 0.067 & 0.060 & 0.067 & 0.170 & 0.190 \\
\hline 4th cumulant & 0.040 & 0.125 & 0.058 & 0.092 & 0.091 & 0.086 & 0.309 & 0.324 & 0.324 & 0.015 & 0.016 & 0.077 & 0.162 & 0.191 \\
\hline Kocherlakota $(t=-0.05)$ & 0.047 & 0.207 & 0.055 & 0.066 & 0.077 & 0.081 & 0.376 & 0.395 & 0.325 & 0.091 & 0.061 & 0.079 & 0.164 & 0.152 \\
\hline Kocherlakota $(t=0.05)$ & 0.043 & 0.216 & 0.057 & 0.063 & 0.076 & 0.080 & 0.405 & 0.417 & 0.342 & 0.088 & 0.060 & 0.081 & 0.168 & 0.159 \\
\hline Kocherlakota $(t=0.125)$ & 0.045 & 0.230 & 0.058 & 0.069 & 0.077 & 0.080 & 0.435 & 0.449 & 0.368 & 0.090 & 0.063 & 0.083 & 0.185 & 0.174 \\
\hline Nass & 0.065 & 0.101 & 0.054 & 0.063 & 0.067 & 0.087 & 0.315 & 0.334 & 0.334 & 0.028 & 0.036 & 0.072 & 0.134 & 0.161 \\
\hline Gupta & 0.048 & 0.137 & 0.061 & 0.075 & 0.078 & 0.055 & 0.099 & 0.108 & 0.088 & 0.019 & 0.021 & 0.093 & 0.107 & 0.097 \\
\hline Baringhaus & 0.040 & 0.179 & 0.051 & 0.051 & 0.057 & 0.072 & 0.355 & 0.374 & 0.303 & 0.096 & 0.064 & 0.063 & 0.144 & 0.132 \\
\hline Nakamura & 0.041 & 0.228 & 0.071 & 0.090 & 0.113 & 0.109 & 0.487 & 0.498 & 0.472 & 0.021 & 0.028 & 0.091 & 0.232 & 0.234 \\
\hline Rueda & 0.045 & 0.219 & 0.058 & 0.065 & 0.075 & 0.084 & 0.421 & 0.440 & 0.374 & 0.108 & 0.080 & 0.080 & 0.177 & 0.169 \\
\hline $\mathrm{KS}$ & 0.053 & 0.197 & 0.067 & 0.071 & 0.078 & 0.085 & 0.370 & 0.390 & 0.320 & 0.100 & 0.073 & 0.075 & 0.157 & 0.160 \\
\hline McIntyre & 0.046 & 0.256 & 0.068 & 0.097 & 0.107 & 0.126 & 0.554 & 0.556 & 0.533 & 0.058 & 0.038 & 0.098 & 0.267 & 0.267 \\
\hline$\chi^{2}$ & 0.048 & 0.147 & 0.068 & 0.060 & 0.067 & 0.065 & 0.260 & 0.290 & 0.223 & 0.076 & 0.060 & 0.064 & 0.112 & 0.106 \\
\hline $\begin{array}{l}\text { Power divergent family } \\
\quad(\lambda=2 / 3)\end{array}$ & 0.044 & 0.133 & 0.062 & 0.056 & 0.063 & 0.059 & 0.250 & 0.282 & 0.210 & 0.073 & 0.056 & 0.061 & 0.102 & 0.099 \\
\hline LRT & 0.050 & 0.312 & 0.076 & 0.117 & 0.132 & 0.138 & 0.574 & 0.589 & 0.550 & 0.007 & 0.012 & 0.116 & 0.307 & 0.291 \\
\hline Hellinger deviance & 0.064 & 0.349 & 0.100 & 0.135 & 0.143 & 0.134 & 0.527 & 0.527 & 0.477 & 0.013 & 0.021 & 0.134 & 0.289 & 0.266 \\
\hline Rayner & 0.050 & 0.181 & 0.075 & 0.093 & 0.113 & 0.122 & 0.461 & 0.445 & 0.463 & 0.008 & 0.010 & 0.095 & 0.220 & 0.242 \\
\hline Cramér-von Mises 1 & 0.053 & 0.153 & 0.053 & 0.066 & 0.064 & 0.066 & 0.340 & 0.327 & 0.296 & 0.081 & 0.071 & 0.063 & 0.128 & 0.125 \\
\hline Cramér-von Mises 2 & 0.053 & 0.190 & 0.059 & 0.077 & 0.075 & 0.077 & 0.418 & 0.408 & 0.379 & 0.076 & 0.064 & 0.072 & 0.171 & 0.167 \\
\hline Cramér-von Mises 3 & 0.053 & 0.175 & 0.056 & 0.071 & 0.070 & 0.072 & 0.382 & 0.371 & 0.341 & 0.083 & 0.070 & 0.065 & 0.155 & 0.150 \\
\hline Cramér-von Mises 4 & 0.053 & 0.180 & 0.058 & 0.077 & 0.074 & 0.075 & 0.390 & 0.375 & 0.344 & 0.069 & 0.060 & 0.071 & 0.156 & 0.150 \\
\hline Rescaled VT & 0.044 & 0.051 & 0.034 & 0.028 & 0.028 & 0.029 & 0.109 & 0.094 & 0.075 & 0.119 & 0.096 & 0.030 & 0.039 & 0.036 \\
\hline $\begin{array}{l}\text { Empirical integrated } \\
\text { distribution function }\end{array}$ & 0.052 & 0.199 & 0.058 & 0.079 & 0.079 & 0.078 & 0.427 & 0.415 & 0.385 & 0.086 & 0.071 & 0.068 & 0.184 & 0.184 \\
\hline $\begin{array}{l}\text { Efron double-exponential } \\
\text { family }\end{array}$ & 0.051 & 0.304 & 0.079 & 0.129 & 0.127 & 0.129 & 0.589 & 0.576 & 0.562 & 0.007 & 0.010 & 0.109 & 0.298 & 0.298 \\
\hline
\end{tabular}

$\dagger$ The Poisson parameter was set to $\lambda=1$. The sample size was 50 ( $\alpha=5 \%, 10000$ replications). 
Table 4. Calculated power for all the tests employed in the simulation study and for all the alternative distributions used $\dagger$

\begin{tabular}{|c|c|c|c|c|c|c|c|c|c|c|c|c|c|c|}
\hline \multirow[t]{2}{*}{ Test } & \multicolumn{14}{|c|}{ Powers for the following alternative distributions: } \\
\hline & (a) & (b) & (c) & (d) & (e) & $(f)$ & (g) & (h) & (i) & (j) & $(k)$ & (l) & (m) & (n) \\
\hline 2nd cumulant & 0.043 & 0.466 & 0.092 & 0.181 & 0.197 & 0.182 & 0.837 & 0.820 & 0.796 & 0.232 & 0.168 & 0.138 & 0.476 & 0.452 \\
\hline VT & 0.053 & 0.519 & 0.109 & 0.211 & 0.232 & 0.212 & 0.866 & 0.846 & 0.817 & 0.201 & 0.141 & 0.171 & 0.515 & 0.493 \\
\hline Böhning & 0.050 & 0.506 & 0.107 & 0.206 & 0.228 & 0.207 & 0.862 & 0.844 & 0.814 & 0.208 & 0.147 & 0.168 & 0.508 & 0.484 \\
\hline Zelterman & 0.046 & 0.478 & 0.098 & 0.188 & 0.201 & 0.189 & 0.843 & 0.829 & 0.809 & 0.232 & 0.168 & 0.150 & 0.486 & 0.457 \\
\hline 3rd cumulant & 0.035 & 0.225 & 0.064 & 0.082 & 0.106 & 0.112 & 0.611 & 0.620 & 0.623 & 0.111 & 0.084 & 0.072 & 0.276 & 0.282 \\
\hline 4th cumulant & 0.053 & 0.138 & 0.069 & 0.089 & 0.097 & 0.086 & 0.426 & 0.438 & 0.452 & 0.025 & 0.022 & 0.072 & 0.190 & 0.218 \\
\hline Kocherlakota $(t=-0.05)$ & 0.051 & 0.332 & 0.049 & 0.086 & 0.108 & 0.100 & 0.645 & 0.623 & 0.529 & 0.112 & 0.099 & 0.097 & 0.264 & 0.245 \\
\hline Kocherlakota $(t=0.05)$ & 0.049 & 0.339 & 0.047 & 0.098 & 0.113 & 0.106 & 0.677 & 0.646 & 0.561 & 0.127 & 0.104 & 0.096 & 0.275 & 0.258 \\
\hline Kocherlakota $(t=0.125)$ & 0.050 & 0.356 & 0.047 & 0.104 & 0.120 & 0.116 & 0.693 & 0.668 & 0.585 & 0.126 & 0.099 & 0.096 & 0.290 & 0.265 \\
\hline Nass & 0.049 & 0.197 & 0.062 & 0.084 & 0.099 & 0.104 & 0.537 & 0.522 & 0.522 & 0.028 & 0.029 & 0.066 & 0.203 & 0.220 \\
\hline Gupta & 0.059 & 0.126 & 0.049 & 0.071 & 0.068 & 0.061 & 0.058 & 0.052 & 0.041 & 0.026 & 0.025 & 0.091 & 0.069 & 0.074 \\
\hline Baringhaus & 0.045 & 0.306 & 0.042 & 0.080 & 0.097 & 0.092 & 0.616 & 0.582 & 0.504 & 0.124 & 0.095 & 0.075 & 0.241 & 0.212 \\
\hline Nakamura & 0.053 & 0.352 & 0.064 & 0.131 & 0.138 & 0.147 & 0.725 & 0.700 & 0.669 & 0.046 & 0.037 & 0.093 & 0.340 & 0.320 \\
\hline Rueda & 0.051 & 0.351 & 0.048 & 0.092 & 0.116 & 0.112 & 0.684 & 0.659 & 0.590 & 0.137 & 0.103 & 0.087 & 0.291 & 0.267 \\
\hline $\mathrm{KS}$ & 0.053 & 0.308 & 0.045 & 0.093 & 0.102 & 0.097 & 0.622 & 0.603 & 0.517 & 0.124 & 0.103 & 0.088 & 0.248 & 0.218 \\
\hline McIntyre & 0.048 & 0.411 & 0.079 & 0.143 & 0.160 & 0.157 & 0.811 & 0.781 & 0.757 & 0.105 & 0.077 & 0.124 & 0.427 & 0.393 \\
\hline$\chi^{2}$ & 0.051 & 0.292 & 0.048 & 0.087 & 0.096 & 0.097 & 0.527 & 0.483 & 0.435 & 0.091 & 0.072 & 0.068 & 0.210 & 0.191 \\
\hline $\begin{array}{l}\text { Power divergent family } \\
\quad(\lambda=2 / 3)\end{array}$ & 0.064 & 0.315 & 0.063 & 0.113 & 0.116 & 0.110 & 0.552 & 0.511 & 0.462 & 0.109 & 0.092 & 0.081 & 0.235 & 0.216 \\
\hline LRT & 0.029 & 0.402 & 0.047 & 0.114 & 0.133 & 0.130 & 0.781 & 0.753 & 0.703 & 0.004 & 0.004 & 0.094 & 0.370 & 0.330 \\
\hline Hellinger deviance & 0.059 & 0.478 & 0.088 & 0.175 & 0.204 & 0.186 & 0.793 & 0.748 & 0.695 & 0.009 & 0.012 & 0.172 & 0.440 & 0.385 \\
\hline Rayner & 0.048 & 0.250 & 0.080 & 0.135 & 0.138 & 0.148 & 0.620 & 0.613 & 0.613 & 0.006 & 0.012 & 0.096 & 0.304 & 0.318 \\
\hline Cramér-von Mises 1 & 0.053 & 0.269 & 0.054 & 0.079 & 0.078 & 0.079 & 0.606 & 0.574 & 0.524 & 0.109 & 0.084 & 0.076 & 0.226 & 0.202 \\
\hline Cramér-von Mises 2 & 0.054 & 0.317 & 0.058 & 0.094 & 0.094 & 0.093 & 0.676 & 0.653 & 0.611 & 0.110 & 0.084 & 0.086 & 0.280 & 0.259 \\
\hline Cramér-von Mises 3 & 0.054 & 0.298 & 0.055 & 0.087 & 0.087 & 0.087 & 0.643 & 0.614 & 0.567 & 0.114 & 0.087 & 0.079 & 0.257 & 0.233 \\
\hline Cramér-von Mises 4 & 0.053 & 0.298 & 0.058 & 0.088 & 0.087 & 0.088 & 0.643 & 0.613 & 0.562 & 0.092 & 0.074 & 0.085 & 0.254 & 0.230 \\
\hline Rescaled VT & 0.045 & 0.135 & 0.033 & 0.032 & 0.033 & 0.033 & 0.356 & 0.325 & 0.266 & 0.191 & 0.142 & 0.035 & 0.105 & 0.086 \\
\hline $\begin{array}{l}\text { Empirical integrated } \\
\text { distribution function }\end{array}$ & 0.054 & 0.342 & 0.060 & 0.103 & 0.104 & 0.104 & 0.672 & 0.650 & 0.599 & 0.127 & 0.094 & 0.087 & 0.308 & 0.293 \\
\hline $\begin{array}{l}\text { Efron double-exponential } \\
\text { family }\end{array}$ & 0.055 & 0.477 & 0.089 & 0.176 & 0.177 & 0.179 & 0.819 & 0.806 & 0.789 & 0.002 & 0.006 & 0.142 & 0.460 & 0.451 \\
\hline
\end{tabular}

$\dagger$ The Poisson parameter was set to $\lambda=1$. The sample size was $100(\alpha=5 \%, 10000$ replications $)$. 
Table 5. Calculated power for all the tests employed in the simulation study and for all the alternative distributions used $\dagger$

\begin{tabular}{|c|c|c|c|c|c|c|c|c|c|c|c|c|c|c|}
\hline \multirow[t]{2}{*}{ Test } & \multicolumn{14}{|c|}{ Powers for the following alternative distributions: } \\
\hline & (a) & (b) & (c) & (d) & (e) & $(f)$ & (g) & (h) & (i) & $(j)$ & ( $k$ ) & (l) & (m) & (n) \\
\hline 2nd cumulant & 0.049 & 0.966 & 0.152 & 0.419 & 0.418 & 0.445 & 1.000 & 1.000 & 1.000 & 0.690 & 0.509 & 0.369 & 0.952 & 0.956 \\
\hline VT & 0.051 & 0.969 & 0.161 & 0.429 & 0.433 & 0.453 & 1.000 & 1.000 & 1.000 & 0.673 & 0.496 & 0.384 & 0.953 & 0.962 \\
\hline Böhning & 0.049 & 0.969 & 0.156 & 0.428 & 0.430 & 0.448 & 1.000 & 1.000 & 1.000 & 0.683 & 0.506 & 0.378 & 0.953 & 0.960 \\
\hline Zelterman & 0.049 & 0.965 & 0.152 & 0.416 & 0.414 & 0.442 & 1.000 & 1.000 & 1.000 & 0.696 & 0.519 & 0.366 & 0.951 & 0.955 \\
\hline 3rd cumulant & 0.054 & 0.748 & 0.105 & 0.240 & 0.240 & 0.245 & 0.999 & 1.000 & 0.997 & 0.500 & 0.306 & 0.179 & 0.828 & 0.827 \\
\hline 4th cumulant & 0.050 & 0.310 & 0.076 & 0.138 & 0.138 & 0.128 & 0.898 & 0.941 & 0.950 & 0.176 & 0.104 & 0.092 & 0.513 & 0.561 \\
\hline Kocherlakota $(t=-0.05)$ & 0.055 & 0.881 & 0.073 & 0.237 & 0.224 & 0.206 & 0.999 & 0.999 & 0.991 & 0.398 & 0.295 & 0.238 & 0.820 & 0.761 \\
\hline Kocherlakota $(t=0.05)$ & 0.053 & 0.896 & 0.077 & 0.249 & 0.244 & 0.233 & 1.000 & 1.000 & 0.994 & 0.416 & 0.307 & 0.251 & 0.854 & 0.804 \\
\hline Kocherlakota $(t=0.125)$ & 0.049 & 0.902 & 0.076 & 0.249 & 0.251 & 0.246 & 1.000 & 1.000 & 0.995 & 0.439 & 0.317 & 0.253 & 0.871 & 0.818 \\
\hline Nass & 0.046 & 0.631 & 0.095 & 0.148 & 0.148 & 0.127 & 0.991 & 0.993 & 0.988 & 0.056 & 0.036 & 0.099 & 0.660 & 0.639 \\
\hline Gupta & 0.062 & 0.014 & 0.041 & 0.035 & 0.029 & 0.019 & 0.000 & 0.000 & 0.000 & 0.055 & 0.049 & 0.050 & 0.009 & 0.007 \\
\hline Baringhaus & 0.054 & 0.884 & 0.076 & 0.237 & 0.227 & 0.217 & 1.000 & 0.999 & 0.993 & 0.407 & 0.302 & 0.234 & 0.837 & 0.776 \\
\hline Nakamura & 0.051 & 0.866 & 0.093 & 0.253 & 0.226 & 0.233 & 1.000 & 0.998 & 0.998 & 0.277 & 0.159 & 0.212 & 0.833 & 0.784 \\
\hline Rueda & 0.047 & 0.908 & 0.071 & 0.256 & 0.261 & 0.247 & 1.000 & 1.000 & 0.995 & 0.459 & 0.328 & 0.248 & 0.881 & 0.834 \\
\hline KS & 0.060 & 0.881 & 0.081 & 0.228 & 0.231 & 0.213 & 1.000 & 0.999 & 0.994 & 0.408 & 0.305 & 0.230 & 0.827 & 0.773 \\
\hline McIntyre & 0.051 & 0.939 & 0.125 & 0.342 & 0.337 & 0.337 & 1.000 & 1.000 & 0.997 & 0.518 & 0.345 & 0.294 & 0.926 & 0.930 \\
\hline$\chi^{2}$ & 0.054 & 0.852 & 0.075 & 0.232 & 0.201 & 0.215 & 1.000 & 0.994 & 0.990 & 0.305 & 0.187 & 0.196 & 0.794 & 0.740 \\
\hline $\begin{array}{l}\text { Power divergent family } \\
\quad(\lambda=2 / 3)\end{array}$ & 0.075 & 0.862 & 0.083 & 0.248 & 0.224 & 0.228 & 1.000 & 0.994 & 0.991 & 0.363 & 0.228 & 0.214 & 0.805 & 0.758 \\
\hline LRT & 0.031 & 0.942 & 0.094 & 0.320 & 0.318 & 0.302 & 1.000 & 1.000 & 0.997 & 0.000 & 0.000 & 0.286 & 0.921 & 0.917 \\
\hline Hellinger deviance & 0.043 & 0.954 & 0.108 & 0.361 & 0.358 & 0.348 & 1.000 & 0.998 & 0.996 & 0.000 & 0.000 & 0.345 & 0.936 & 0.897 \\
\hline Rayner & 0.045 & 0.414 & 0.107 & 0.169 & 0.172 & 0.170 & 0.958 & 0.965 & 0.972 & 0.003 & 0.005 & 0.115 & 0.574 & 0.618 \\
\hline Cramér-von Mises 1 & 0.050 & 0.886 & 0.071 & 0.209 & 0.206 & 0.199 & 0.999 & 0.998 & 0.996 & 0.365 & 0.248 & 0.195 & 0.792 & 0.743 \\
\hline Cramér-von Mises 2 & 0.049 & 0.914 & 0.081 & 0.248 & 0.244 & 0.238 & 1.000 & 0.999 & 0.998 & 0.412 & 0.273 & 0.220 & 0.846 & 0.812 \\
\hline Cramér-von Mises 3 & 0.051 & 0.904 & 0.077 & 0.234 & 0.230 & 0.224 & 0.999 & 0.999 & 0.997 & 0.401 & 0.270 & 0.207 & 0.826 & 0.784 \\
\hline Cramér-von Mises 4 & 0.050 & 0.895 & 0.075 & 0.221 & 0.216 & 0.212 & 0.999 & 0.999 & 0.996 & 0.347 & 0.233 & 0.205 & 0.806 & 0.759 \\
\hline Rescaled VT & 0.047 & 0.905 & 0.059 & 0.213 & 0.215 & 0.213 & 0.999 & 0.999 & 0.998 & 0.633 & 0.451 & 0.183 & 0.846 & 0.820 \\
\hline $\begin{array}{l}\text { Empirical integrated } \\
\text { distribution function }\end{array}$ & 0.052 & 0.924 & 0.090 & 0.285 & 0.282 & 0.282 & 0.998 & 0.998 & 0.994 & 0.475 & 0.324 & 0.237 & 0.864 & 0.829 \\
\hline $\begin{array}{l}\text { Efron double-exponential } \\
\text { family }\end{array}$ & 0.055 & 0.964 & 0.158 & 0.439 & 0.440 & 0.436 & 1.000 & 1.000 & 1.000 & 0.000 & 0.000 & 0.342 & 0.947 & 0.937 \\
\hline
\end{tabular}

$\dagger$ The Poisson parameter was set to $\lambda=1$. The sample size was 500 ( $\alpha=5 \%, 10000$ replications $)$. 
Table 6. Calculated power for all the tests employed in the simulation study and for all the alternative distributions used $\dagger$

\begin{tabular}{|c|c|c|c|c|c|c|c|c|c|c|c|c|c|c|}
\hline \multirow[t]{2}{*}{ Test } & \multicolumn{14}{|c|}{ Powers for the following alternative distributions: } \\
\hline & (a) & (b) & (c) & (d) & (e) & $(f)$ & (g) & (h) & (i) & (j) & $(k)$ & (l) & (m) & (n) \\
\hline 2nd cumulant & 0.061 & 0.885 & 0.154 & 0.145 & 0.118 & 0.125 & 0.661 & 0.634 & 0.641 & 0.287 & 0.110 & 0.326 & 0.840 & 0.827 \\
\hline VT & 0.072 & 0.899 & 0.177 & 0.157 & 0.137 & 0.149 & 0.687 & 0.653 & 0.673 & 0.265 & 0.099 & 0.346 & 0.855 & 0.841 \\
\hline Böhning & 0.072 & 0.899 & 0.177 & 0.157 & 0.137 & 0.149 & 0.687 & 0.653 & 0.673 & 0.253 & 0.087 & 0.346 & 0.855 & 0.841 \\
\hline Zelterman & 0.058 & 0.882 & 0.150 & 0.141 & 0.117 & 0.121 & 0.654 & 0.624 & 0.635 & 0.288 & 0.110 & 0.315 & 0.839 & 0.816 \\
\hline 3rd cumulant & 0.053 & 0.407 & 0.089 & 0.090 & 0.090 & 0.077 & 0.375 & 0.344 & 0.408 & 0.067 & 0.050 & 0.114 & 0.549 & 0.596 \\
\hline 4th cumulant & 0.046 & 0.507 & 0.106 & 0.106 & 0.089 & 0.091 & 0.328 & 0.287 & 0.335 & 0.006 & 0.022 & 0.181 & 0.442 & 0.455 \\
\hline Kocherlakota $(t=-0.05)$ & 0.055 & 0.502 & 0.048 & 0.074 & 0.058 & 0.068 & 0.213 & 0.212 & 0.207 & 0.062 & 0.045 & 0.131 & 0.404 & 0.341 \\
\hline Kocherlakota $(t=0.05)$ & 0.048 & 0.578 & 0.053 & 0.070 & 0.058 & 0.064 & 0.271 & 0.244 & 0.237 & 0.082 & 0.044 & 0.147 & 0.463 & 0.399 \\
\hline Kocherlakota $(t=0.125)$ & 0.049 & 0.640 & 0.060 & 0.080 & 0.064 & 0.063 & 0.299 & 0.275 & 0.279 & 0.115 & 0.049 & 0.162 & 0.526 & 0.441 \\
\hline Nass & 0.065 & 0.482 & 0.087 & 0.069 & 0.079 & 0.074 & 0.338 & 0.298 & 0.357 & 0.023 & 0.032 & 0.109 & 0.498 & 0.539 \\
\hline Gupta & 0.058 & 0.511 & 0.073 & 0.095 & 0.072 & 0.097 & 0.219 & 0.202 & 0.173 & 0.008 & 0.017 & 0.199 & 0.238 & 0.176 \\
\hline Baringhaus & 0.048 & 0.732 & 0.071 & 0.092 & 0.074 & 0.073 & 0.387 & 0.370 & 0.358 & 0.092 & 0.032 & 0.208 & 0.631 & 0.577 \\
\hline Nakamura & 0.050 & 0.808 & 0.109 & 0.102 & 0.094 & 0.084 & 0.492 & 0.453 & 0.478 & 0.055 & 0.035 & 0.210 & 0.704 & 0.688 \\
\hline Rueda & 0.050 & 0.769 & 0.071 & 0.098 & 0.066 & 0.075 & 0.410 & 0.394 & 0.385 & 0.113 & 0.038 & 0.225 & 0.650 & 0.606 \\
\hline KS & 0.050 & 0.668 & 0.070 & 0.077 & 0.057 & 0.063 & 0.306 & 0.276 & 0.274 & 0.117 & 0.060 & 0.128 & 0.524 & 0.472 \\
\hline McIntyre & 0.054 & 0.857 & 0.124 & 0.124 & 0.096 & 0.097 & 0.600 & 0.577 & 0.597 & 0.126 & 0.040 & 0.273 & 0.811 & 0.774 \\
\hline$\chi^{2}$ & 0.065 & 0.682 & 0.074 & 0.080 & 0.067 & 0.074 & 0.292 & 0.289 & 0.267 & 0.075 & 0.050 & 0.130 & 0.514 & 0.461 \\
\hline $\begin{array}{l}\text { Power divergent family } \\
\quad(\lambda=2 / 3)\end{array}$ & 0.058 & 0.655 & 0.069 & 0.076 & 0.059 & 0.067 & 0.273 & 0.268 & 0.243 & 0.070 & 0.053 & 0.116 & 0.484 & 0.430 \\
\hline LRT & 0.036 & 0.868 & 0.115 & 0.123 & 0.088 & 0.093 & 0.564 & 0.534 & 0.555 & 0.001 & 0.012 & 0.269 & 0.796 & 0.755 \\
\hline Hellinger deviance & 0.065 & 0.900 & 0.133 & 0.152 & 0.110 & 0.116 & 0.562 & 0.556 & 0.543 & 0.003 & 0.016 & 0.348 & 0.776 & 0.700 \\
\hline Rayner & 0.051 & 0.782 & 0.126 & 0.121 & 0.105 & 0.091 & 0.507 & 0.464 & 0.511 & 0.050 & 0.025 & 0.201 & 0.714 & 0.706 \\
\hline Cramér-von Mises 1 & 0.049 & 0.688 & 0.065 & 0.062 & 0.061 & 0.062 & 0.299 & 0.296 & 0.288 & 0.127 & 0.066 & 0.145 & 0.516 & 0.483 \\
\hline Cramér-von Mises 2 & 0.050 & 0.816 & 0.087 & 0.077 & 0.074 & 0.076 & 0.445 & 0.436 & 0.427 & 0.127 & 0.058 & 0.206 & 0.688 & 0.652 \\
\hline Cramér-von Mises 3 & 0.049 & 0.794 & 0.079 & 0.073 & 0.070 & 0.072 & 0.407 & 0.398 & 0.389 & 0.132 & 0.062 & 0.188 & 0.646 & 0.603 \\
\hline Cramér-von Mises 4 & 0.048 & 0.707 & 0.074 & 0.068 & 0.065 & 0.067 & 0.326 & 0.321 & 0.316 & 0.105 & 0.056 & 0.156 & 0.553 & 0.517 \\
\hline Rescaled VT & 0.044 & 0.508 & 0.024 & 0.025 & 0.023 & 0.025 & 0.128 & 0.120 & 0.107 & 0.257 & 0.104 & 0.052 & 0.276 & 0.214 \\
\hline $\begin{array}{l}\text { Empirical integrated } \\
\text { distribution function }\end{array}$ & 0.052 & 0.840 & 0.095 & 0.083 & 0.080 & 0.081 & 0.494 & 0.489 & 0.483 & 0.175 & 0.070 & 0.218 & 0.727 & 0.694 \\
\hline $\begin{array}{l}\text { Efron double-exponential } \\
\text { family }\end{array}$ & 0.054 & 0.740 & 0.135 & 0.110 & 0.111 & 0.109 & 0.488 & 0.493 & 0.496 & 0.002 & 0.015 & 0.210 & 0.676 & 0.679 \\
\hline
\end{tabular}

$\dagger$ The Poisson parameter was set to $\lambda=3$. The sample size was 50 ( $\alpha=5 \%, 10000$ replications). 
Table 7. Calculated power for all the tests employed in the simulation study and for all the alternative distributions used $\dagger$

\begin{tabular}{|c|c|c|c|c|c|c|c|c|c|c|c|c|c|c|}
\hline \multirow[t]{2}{*}{ Test } & \multicolumn{14}{|c|}{ Powers for the following alternative distributions: } \\
\hline & (a) & (b) & (c) & (d) & (e) & $(f)$ & (g) & (h) & (i) & $(j)$ & ( $k$ ) & (l) & (m) & (n) \\
\hline 2nd cumulant & 0.063 & 0.990 & 0.214 & 0.182 & 0.178 & 0.166 & 0.869 & 0.873 & 0.856 & 0.496 & 0.172 & 0.513 & 0.975 & 0.974 \\
\hline VT & 0.072 & 0.991 & 0.241 & 0.209 & 0.191 & 0.183 & 0.879 & 0.887 & 0.869 & 0.458 & 0.151 & 0.541 & 0.980 & 0.977 \\
\hline Böhning & 0.071 & 0.991 & 0.241 & 0.207 & 0.190 & 0.183 & 0.879 & 0.887 & 0.869 & 0.453 & 0.147 & 0.541 & 0.980 & 0.977 \\
\hline Zelterman & 0.063 & 0.990 & 0.210 & 0.177 & 0.177 & 0.162 & 0.865 & 0.873 & 0.855 & 0.495 & 0.171 & 0.510 & 0.974 & 0.970 \\
\hline 3rd cumulant & 0.041 & 0.681 & 0.122 & 0.101 & 0.089 & 0.081 & 0.541 & 0.565 & 0.585 & 0.151 & 0.065 & 0.145 & 0.815 & 0.819 \\
\hline 4th cumulant & 0.049 & 0.448 & 0.116 & 0.105 & 0.095 & 0.082 & 0.335 & 0.347 & 0.368 & 0.005 & 0.022 & 0.171 & 0.538 & 0.581 \\
\hline Kocherlakota $(t=-0.05)$ & 0.042 & 0.741 & 0.064 & 0.074 & 0.070 & 0.054 & 0.378 & 0.403 & 0.296 & 0.091 & 0.052 & 0.210 & 0.653 & 0.520 \\
\hline Kocherlakota $(t=0.05)$ & 0.038 & 0.824 & 0.068 & 0.078 & 0.070 & 0.060 & 0.452 & 0.466 & 0.364 & 0.132 & 0.059 & 0.249 & 0.718 & 0.607 \\
\hline Kocherlakota $(t=0.125)$ & 0.038 & 0.884 & 0.067 & 0.079 & 0.073 & 0.063 & 0.499 & 0.526 & 0.420 & 0.158 & 0.062 & 0.282 & 0.781 & 0.667 \\
\hline Nass & 0.050 & 0.761 & 0.101 & 0.088 & 0.076 & 0.064 & 0.463 & 0.467 & 0.483 & 0.011 & 0.031 & 0.126 & 0.778 & 0.755 \\
\hline Gupta & 0.051 & 0.447 & 0.079 & 0.092 & 0.087 & 0.086 & 0.154 & 0.144 & 0.123 & 0.007 & 0.027 & 0.206 & 0.126 & 0.098 \\
\hline Baringhaus & 0.039 & 0.940 & 0.083 & 0.093 & 0.085 & 0.069 & 0.589 & 0.625 & 0.552 & 0.145 & 0.047 & 0.325 & 0.875 & 0.804 \\
\hline Nakamura & 0.052 & 0.968 & 0.149 & 0.132 & 0.114 & 0.106 & 0.702 & 0.690 & 0.697 & 0.147 & 0.062 & 0.326 & 0.922 & 0.900 \\
\hline Rueda & 0.042 & 0.955 & 0.093 & 0.102 & 0.092 & 0.083 & 0.659 & 0.680 & 0.606 & 0.198 & 0.061 & 0.381 & 0.907 & 0.845 \\
\hline $\mathrm{KS}$ & 0.052 & 0.920 & 0.090 & 0.095 & 0.076 & 0.069 & 0.515 & 0.500 & 0.502 & 0.197 & 0.076 & 0.221 & 0.799 & 0.751 \\
\hline McIntyre & 0.050 & 0.978 & 0.184 & 0.147 & 0.148 & 0.123 & 0.826 & 0.847 & 0.812 & 0.291 & 0.069 & 0.444 & 0.965 & 0.963 \\
\hline$\chi^{2}$ & 0.069 & 0.936 & 0.097 & 0.093 & 0.087 & 0.073 & 0.550 & 0.551 & 0.505 & 0.120 & 0.050 & 0.240 & 0.822 & 0.759 \\
\hline $\begin{array}{l}\text { Power divergent family } \\
\quad(\lambda=2 / 3)\end{array}$ & 0.062 & 0.929 & 0.081 & 0.079 & 0.078 & 0.066 & 0.517 & 0.519 & 0.469 & 0.122 & 0.048 & 0.225 & 0.803 & 0.721 \\
\hline LRT & 0.040 & 0.981 & 0.152 & 0.127 & 0.123 & 0.096 & 0.806 & 0.823 & 0.773 & 0.000 & 0.003 & 0.423 & 0.961 & 0.953 \\
\hline Hellinger deviance & 0.062 & 0.990 & 0.174 & 0.164 & 0.144 & 0.157 & 0.797 & 0.804 & 0.766 & 0.001 & 0.009 & 0.526 & 0.953 & 0.918 \\
\hline Rayner & 0.058 & 0.959 & 0.170 & 0.140 & 0.123 & 0.109 & 0.709 & 0.738 & 0.719 & 0.107 & 0.042 & 0.292 & 0.931 & 0.916 \\
\hline Cramér-von Mises 1 & 0.050 & 0.944 & 0.081 & 0.072 & 0.074 & 0.075 & 0.548 & 0.532 & 0.516 & 0.220 & 0.080 & 0.248 & 0.818 & 0.779 \\
\hline Cramér-von Mises 2 & 0.052 & 0.980 & 0.110 & 0.095 & 0.096 & 0.094 & 0.706 & 0.693 & 0.677 & 0.252 & 0.079 & 0.343 & 0.921 & 0.897 \\
\hline Cramér-von Mises 3 & 0.052 & 0.975 & 0.099 & 0.088 & 0.090 & 0.089 & 0.674 & 0.656 & 0.638 & 0.249 & 0.082 & 0.320 & 0.903 & 0.870 \\
\hline Cramér-von Mises 4 & 0.052 & 0.951 & 0.090 & 0.080 & 0.081 & 0.080 & 0.574 & 0.559 & 0.545 & 0.194 & 0.071 & 0.261 & 0.841 & 0.804 \\
\hline Rescaled VT & 0.044 & 0.944 & 0.039 & 0.037 & 0.035 & 0.037 & 0.498 & 0.476 & 0.442 & 0.450 & 0.140 & 0.191 & 0.786 & 0.704 \\
\hline $\begin{array}{l}\text { Empirical integrated } \\
\text { distribution function }\end{array}$ & 0.055 & 0.982 & 0.126 & 0.105 & 0.108 & 0.106 & 0.736 & 0.727 & 0.709 & 0.333 & 0.101 & 0.374 & 0.914 & 0.884 \\
\hline $\begin{array}{l}\text { Efron double-exponential } \\
\text { family }\end{array}$ & 0.058 & 0.941 & 0.180 & 0.147 & 0.149 & 0.146 & 0.712 & 0.710 & 0.717 & 0.000 & 0.010 & 0.321 & 0.892 & 0.888 \\
\hline
\end{tabular}

$\dagger$ The Poisson parameter was set to $\lambda=3$. The sample size was $100(\alpha=5 \%, 10000$ replications). 
Table 8. Calculated power for all the tests employed in the simulation study and for all the alternative distributions used $\dagger$

\begin{tabular}{|c|c|c|c|c|c|c|c|c|c|c|c|c|c|c|}
\hline \multirow[t]{2}{*}{ Test } & \multicolumn{14}{|c|}{ Powers for the following alternative distributions: } \\
\hline & (a) & (b) & (c) & (d) & (e) & $(f)$ & (g) & (h) & (i) & $(j)$ & $(k)$ & (l) & (m) & $(n)$ \\
\hline 2nd cumulant & 0.073 & 1.000 & 0.575 & 0.479 & 0.453 & 0.460 & 1.000 & 1.000 & 1.000 & 0.989 & 0.512 & 0.983 & 1.000 & 1.000 \\
\hline VT & 0.078 & 1.000 & 0.582 & 0.488 & 0.464 & 0.470 & 1.000 & 1.000 & 1.000 & 0.988 & 0.507 & 0.984 & 1.000 & 1.000 \\
\hline Böhning & 0.079 & 1.000 & 0.585 & 0.490 & 0.466 & 0.470 & 1.000 & 1.000 & 1.000 & 0.989 & 0.508 & 0.984 & 1.000 & 1.000 \\
\hline Zelterman & 0.071 & 1.000 & 0.563 & 0.467 & 0.447 & 0.451 & 1.000 & 1.000 & 1.000 & 0.990 & 0.526 & 0.979 & 1.000 & 1.000 \\
\hline 3rd cumulant & 0.053 & 1.000 & 0.325 & 0.221 & 0.191 & 0.209 & 0.997 & 0.994 & 0.990 & 0.765 & 0.182 & 0.522 & 1.000 & 0.999 \\
\hline 4 th cumulant & 0.054 & 0.440 & 0.157 & 0.128 & 0.093 & 0.103 & 0.703 & 0.755 & 0.787 & 0.018 & 0.033 & 0.176 & 0.903 & 0.959 \\
\hline Kocherlakota $(t=-0.05)$ & 0.055 & 1.000 & 0.116 & 0.122 & 0.119 & 0.117 & 0.943 & 0.922 & 0.890 & 0.393 & 0.135 & 0.662 & 0.999 & 0.990 \\
\hline Kocherlakota $(t=0.05)$ & 0.053 & 1.000 & 0.145 & 0.141 & 0.157 & 0.134 & 0.986 & 0.973 & 0.941 & 0.521 & 0.173 & 0.773 & 1.000 & 0.999 \\
\hline Kocherlakota $(t=0.125)$ & 0.054 & 1.000 & 0.169 & 0.153 & 0.175 & 0.150 & 0.995 & 0.986 & 0.963 & 0.612 & 0.196 & 0.824 & 1.000 & 1.000 \\
\hline Nass & 0.054 & 1.000 & 0.182 & 0.116 & 0.100 & 0.122 & 0.992 & 0.991 & 0.991 & 0.147 & 0.014 & 0.502 & 1.000 & 1.000 \\
\hline Gupta & 0.050 & 0.263 & 0.019 & 0.053 & 0.047 & 0.058 & 0.013 & 0.006 & 0.007 & 0.007 & 0.040 & 0.140 & 0.006 & 0.003 \\
\hline Baringhaus & 0.057 & 1.000 & 0.223 & 0.196 & 0.210 & 0.187 & 1.000 & 1.000 & 0.998 & 0.741 & 0.221 & 0.906 & 1.000 & 1.000 \\
\hline Nakamura & 0.054 & 1.000 & 0.319 & 0.246 & 0.215 & 0.238 & 1.000 & 1.000 & 1.000 & 0.842 & 0.201 & 0.912 & 1.000 & 1.000 \\
\hline Rueda & 0.062 & 1.000 & 0.241 & 0.217 & 0.223 & 0.216 & 1.000 & 1.000 & 0.999 & 0.797 & 0.246 & 0.930 & 1.000 & 1.000 \\
\hline KS & 0.044 & 1.000 & 0.223 & 0.157 & 0.152 & 0.175 & 0.999 & 0.996 & 0.997 & 0.709 & 0.194 & 0.838 & 1.000 & 1.000 \\
\hline McIntyre & 0.059 & 1.000 & 0.480 & 0.396 & 0.353 & 0.382 & 1.000 & 1.000 & 1.000 & 0.963 & 0.360 & 0.965 & 1.000 & 1.000 \\
\hline$\chi^{2}$ & 0.053 & 1.000 & 0.290 & 0.206 & 0.201 & 0.228 & 1.000 & 0.999 & 0.999 & 0.673 & 0.145 & 0.861 & 1.000 & 1.000 \\
\hline $\begin{array}{l}\text { Power divergent family } \\
\quad(\lambda=2 / 3)\end{array}$ & 0.048 & 1.000 & 0.264 & 0.186 & 0.186 & 0.199 & 1.000 & 0.999 & 0.999 & 0.694 & 0.152 & 0.844 & 1.000 & 1.000 \\
\hline LRT & 0.049 & 1.000 & 0.495 & 0.413 & 0.374 & 0.399 & 1.000 & 1.000 & 1.000 & 0.000 & 0.000 & 0.974 & 1.000 & 1.000 \\
\hline Hellinger deviance & 0.042 & 1.000 & 0.438 & 0.355 & 0.352 & 0.352 & 1.000 & 1.000 & 1.000 & 0.000 & 0.000 & 0.970 & 1.000 & 1.000 \\
\hline Rayner & 0.066 & 1.000 & 0.330 & 0.243 & 0.230 & 0.231 & 1.000 & 1.000 & 1.000 & 0.778 & 0.135 & 0.883 & 1.000 & 1.000 \\
\hline Cramér-von Mises 1 & 0.051 & 1.000 & 0.231 & 0.184 & 0.182 & 0.180 & 0.998 & 0.998 & 0.997 & 0.819 & 0.214 & 0.857 & 1.000 & 1.000 \\
\hline Cramér-von Mises 2 & 0.051 & 1.000 & 0.320 & 0.252 & 0.253 & 0.245 & 1.000 & 1.000 & 1.000 & 0.907 & 0.264 & 0.933 & 1.000 & 1.000 \\
\hline Cramér-von Mises 3 & 0.050 & 1.000 & 0.291 & 0.235 & 0.235 & 0.229 & 0.999 & 1.000 & 1.000 & 0.892 & 0.254 & 0.922 & 1.000 & 1.000 \\
\hline Cramér-von Mises 4 & 0.051 & 1.000 & 0.241 & 0.193 & 0.191 & 0.188 & 0.998 & 0.998 & 0.997 & 0.805 & 0.202 & 0.866 & 1.000 & 1.000 \\
\hline Rescaled VT & 0.048 & 1.000 & 0.325 & 0.249 & 0.250 & 0.243 & 1.000 & 1.000 & 1.000 & 0.981 & 0.434 & 0.943 & 1.000 & 1.000 \\
\hline $\begin{array}{l}\text { Empirical integrated } \\
\text { distribution function }\end{array}$ & 0.051 & 1.000 & 0.373 & 0.301 & 0.296 & 0.299 & 1.000 & 1.000 & 0.999 & 0.946 & 0.331 & 0.949 & 1.000 & 1.000 \\
\hline $\begin{array}{l}\text { Efron double-exponential } \\
\text { family }\end{array}$ & 0.061 & 1.000 & 0.444 & 0.339 & 0.339 & 0.337 & 0.998 & 0.999 & 0.999 & 0.000 & 0.001 & 0.807 & 1.000 & 1.000 \\
\hline
\end{tabular}

$\dagger$ The Poisson parameter was set to $\lambda=3$. The sample size was $500(\alpha=5 \%, 10000$ replications $)$. 
Table 9. Calculated power for all the tests employed in the simulation study and for all the alternative distributions used $\dagger$

\begin{tabular}{|c|c|c|c|c|c|c|c|c|c|c|c|c|c|c|}
\hline \multirow[t]{2}{*}{ Test } & \multicolumn{14}{|c|}{ Powers for the following alternative distributions: } \\
\hline & (a) & (b) & (c) & (d) & (e) & $(f)$ & (g) & (h) & (i) & $(j)$ & ( $k$ ) & (l) & (m) & (n) \\
\hline 2nd cumulant & 0.068 & 0.996 & 0.259 & 0.135 & 0.130 & 0.130 & 0.631 & 0.623 & 0.631 & 0.384 & 0.118 & 0.561 & 0.986 & 0.979 \\
\hline VT & 0.086 & 0.996 & 0.290 & 0.155 & 0.155 & 0.146 & 0.672 & 0.655 & 0.672 & 0.364 & 0.099 & 0.621 & 0.988 & 0.983 \\
\hline Böhning & 0.087 & 0.996 & 0.291 & 0.157 & 0.157 & 0.148 & 0.674 & 0.659 & 0.673 & 0.355 & 0.097 & 0.626 & 0.989 & 0.983 \\
\hline Zelterman & 0.068 & 0.996 & 0.258 & 0.135 & 0.130 & 0.127 & 0.629 & 0.621 & 0.628 & 0.384 & 0.118 & 0.560 & 0.986 & 0.979 \\
\hline 3rd cumulant & 0.052 & 0.619 & 0.144 & 0.077 & 0.073 & 0.073 & 0.311 & 0.309 & 0.339 & 0.036 & 0.066 & 0.187 & 0.786 & 0.815 \\
\hline 4th cumulant & 0.064 & 0.768 & 0.163 & 0.096 & 0.095 & 0.087 & 0.324 & 0.319 & 0.333 & 0.002 & 0.031 & 0.328 & 0.653 & 0.617 \\
\hline Kocherlakota $(t=-0.05)$ & 0.039 & 0.570 & 0.101 & 0.072 & 0.064 & 0.063 & 0.204 & 0.205 & 0.167 & 0.009 & 0.026 & 0.247 & 0.506 & 0.382 \\
\hline Kocherlakota $(t=0.05)$ & 0.042 & 0.598 & 0.073 & 0.049 & 0.058 & 0.046 & 0.162 & 0.141 & 0.122 & 0.058 & 0.049 & 0.182 & 0.523 & 0.382 \\
\hline Kocherlakota $(t=0.125)$ & 0.041 & 0.699 & 0.070 & 0.044 & 0.056 & 0.044 & 0.187 & 0.151 & 0.134 & 0.101 & 0.054 & 0.200 & 0.589 & 0.464 \\
\hline Nass & 0.058 & 0.874 & 0.136 & 0.071 & 0.078 & 0.080 & 0.303 & 0.307 & 0.341 & 0.016 & 0.039 & 0.201 & 0.848 & 0.815 \\
\hline Gupta & 0.056 & 0.759 & 0.122 & 0.092 & 0.090 & 0.076 & 0.266 & 0.250 & 0.226 & 0.000 & 0.021 & 0.392 & 0.307 & 0.232 \\
\hline Baringhaus & 0.052 & 0.988 & 0.136 & 0.095 & 0.091 & 0.079 & 0.460 & 0.434 & 0.413 & 0.072 & 0.045 & 0.480 & 0.934 & 0.921 \\
\hline Nakamura & 0.066 & 0.992 & 0.178 & 0.085 & 0.089 & 0.093 & 0.461 & 0.437 & 0.469 & 0.079 & 0.060 & 0.427 & 0.942 & 0.929 \\
\hline Rueda & 0.046 & 0.984 & 0.118 & 0.089 & 0.090 & 0.071 & 0.427 & 0.403 & 0.374 & 0.068 & 0.037 & 0.468 & 0.923 & 0.897 \\
\hline KS & 0.063 & 0.955 & 0.098 & 0.069 & 0.055 & 0.061 & 0.261 & 0.269 & 0.278 & 0.132 & 0.069 & 0.272 & 0.778 & 0.769 \\
\hline McIntyre & 0.066 & 0.995 & 0.216 & 0.110 & 0.108 & 0.111 & 0.587 & 0.570 & 0.589 & 0.171 & 0.046 & 0.509 & 0.976 & 0.974 \\
\hline$\chi^{2}$ & 0.048 & 0.961 & 0.098 & 0.068 & 0.075 & 0.068 & 0.303 & 0.284 & 0.280 & 0.064 & 0.048 & 0.305 & 0.821 & 0.782 \\
\hline $\begin{array}{l}\text { Power divergent family } \\
\quad(\lambda=2 / 3)\end{array}$ & 0.051 & 0.955 & 0.099 & 0.068 & 0.076 & 0.071 & 0.285 & 0.273 & 0.266 & 0.075 & 0.050 & 0.294 & 0.809 & 0.760 \\
\hline LRT & 0.055 & 0.996 & 0.202 & 0.101 & 0.097 & 0.095 & 0.577 & 0.539 & 0.549 & 0.000 & 0.013 & 0.529 & 0.970 & 0.966 \\
\hline Hellinger deviance & 0.054 & 0.993 & 0.153 & 0.083 & 0.087 & 0.077 & 0.484 & 0.450 & 0.442 & 0.001 & 0.013 & 0.495 & 0.915 & 0.873 \\
\hline Rayner & 0.055 & 0.991 & 0.194 & 0.112 & 0.100 & 0.099 & 0.513 & 0.490 & 0.503 & 0.042 & 0.042 & 0.431 & 0.952 & 0.944 \\
\hline Cramér-von Mises 1 & 0.050 & 0.967 & 0.095 & 0.061 & 0.062 & 0.062 & 0.295 & 0.294 & 0.289 & 0.162 & 0.064 & 0.302 & 0.817 & 0.786 \\
\hline Cramér-von Mises 2 & 0.053 & 0.992 & 0.142 & 0.079 & 0.078 & 0.077 & 0.450 & 0.447 & 0.444 & 0.171 & 0.059 & 0.433 & 0.936 & 0.913 \\
\hline Cramér-von Mises 3 & 0.052 & 0.989 & 0.126 & 0.073 & 0.071 & 0.072 & 0.413 & 0.407 & 0.403 & 0.174 & 0.062 & 0.402 & 0.915 & 0.885 \\
\hline Cramér-von Mises 4 & 0.052 & 0.968 & 0.106 & 0.065 & 0.066 & 0.065 & 0.309 & 0.308 & 0.308 & 0.138 & 0.057 & 0.302 & 0.832 & 0.801 \\
\hline Rescaled VT & 0.040 & 0.913 & 0.021 & 0.023 & 0.022 & 0.023 & 0.126 & 0.120 & 0.109 & 0.328 & 0.102 & 0.152 & 0.588 & 0.465 \\
\hline $\begin{array}{l}\text { Empirical integrated } \\
\text { distribution function }\end{array}$ & 0.054 & 0.993 & 0.157 & 0.081 & 0.083 & 0.080 & 0.504 & 0.502 & 0.497 & 0.231 & 0.070 & 0.460 & 0.952 & 0.933 \\
\hline $\begin{array}{l}\text { Efron double-exponential } \\
\text { family }\end{array}$ & 0.056 & 0.989 & 0.223 & 0.122 & 0.119 & 0.118 & 0.575 & 0.575 & 0.578 & 0.001 & 0.014 & 0.513 & 0.947 & 0.937 \\
\hline
\end{tabular}

$\dagger$ The Poisson parameter was set to $\lambda=5$. The sample size was 50 ( $\alpha=5 \%, 10000$ replications). 
Table 10. Calculated power for all the tests employed in the simulation study and for all the alternative distributions used $\dagger$

\begin{tabular}{|c|c|c|c|c|c|c|c|c|c|c|c|c|c|c|}
\hline \multirow[t]{2}{*}{ Test } & \multicolumn{14}{|c|}{ Powers for the following alternative distributions: } \\
\hline & (a) & (b) & (c) & (d) & (e) & $(f)$ & (g) & (h) & (i) & (j) & $(k)$ & (l) & (m) & (n) \\
\hline 2nd cumulant & 0.052 & 1.000 & 0.344 & 0.162 & 0.168 & 0.171 & 0.864 & 0.879 & 0.867 & 0.634 & 0.169 & 0.820 & 1.000 & 0.998 \\
\hline VT & 0.068 & 1.000 & 0.382 & 0.193 & 0.192 & 0.204 & 0.890 & 0.896 & 0.879 & 0.597 & 0.144 & 0.844 & 1.000 & 0.998 \\
\hline Böhning & 0.064 & 1.000 & 0.375 & 0.191 & 0.183 & 0.195 & 0.884 & 0.893 & 0.874 & 0.597 & 0.144 & 0.840 & 1.000 & 0.998 \\
\hline Zelterman & 0.057 & 1.000 & 0.354 & 0.168 & 0.172 & 0.179 & 0.869 & 0.883 & 0.869 & 0.631 & 0.168 & 0.825 & 1.000 & 0.998 \\
\hline 3rd cumulant & 0.065 & 0.871 & 0.195 & 0.096 & 0.087 & 0.097 & 0.490 & 0.519 & 0.545 & 0.110 & 0.043 & 0.226 & 0.947 & 0.964 \\
\hline 4th cumulant & 0.052 & 0.786 & 0.157 & 0.105 & 0.091 & 0.104 & 0.348 & 0.388 & 0.380 & 0.000 & 0.015 & 0.343 & 0.748 & 0.771 \\
\hline Kocherlakota $(t=-0.05)$ & 0.054 & 0.714 & 0.089 & 0.066 & 0.085 & 0.088 & 0.244 & 0.232 & 0.227 & 0.009 & 0.033 & 0.278 & 0.700 & 0.549 \\
\hline Kocherlakota $(t=0.05)$ & 0.048 & 0.826 & 0.056 & 0.052 & 0.064 & 0.065 & 0.247 & 0.235 & 0.205 & 0.086 & 0.049 & 0.284 & 0.795 & 0.635 \\
\hline Kocherlakota $(t=0.125)$ & 0.044 & 0.908 & 0.056 & 0.048 & 0.063 & 0.070 & 0.287 & 0.284 & 0.244 & 0.157 & 0.059 & 0.336 & 0.877 & 0.732 \\
\hline Nass & 0.056 & 0.991 & 0.144 & 0.080 & 0.085 & 0.084 & 0.451 & 0.466 & 0.464 & 0.006 & 0.017 & 0.282 & 0.971 & 0.966 \\
\hline Gupta & 0.050 & 0.749 & 0.100 & 0.088 & 0.087 & 0.088 & 0.202 & 0.208 & 0.169 & 0.002 & 0.018 & 0.411 & 0.191 & 0.094 \\
\hline Baringhaus & 0.055 & 1.000 & 0.164 & 0.089 & 0.106 & 0.112 & 0.680 & 0.683 & 0.646 & 0.188 & 0.045 & 0.720 & 0.998 & 0.997 \\
\hline Nakamura & 0.050 & 1.000 & 0.218 & 0.095 & 0.110 & 0.111 & 0.686 & 0.718 & 0.706 & 0.201 & 0.054 & 0.673 & 0.999 & 0.997 \\
\hline Rueda & 0.056 & 1.000 & 0.157 & 0.086 & 0.108 & 0.106 & 0.658 & 0.659 & 0.630 & 0.204 & 0.048 & 0.715 & 0.995 & 0.994 \\
\hline KS & 0.045 & 1.000 & 0.117 & 0.059 & 0.061 & 0.073 & 0.468 & 0.524 & 0.483 & 0.216 & 0.074 & 0.496 & 0.977 & 0.964 \\
\hline McIntyre & 0.056 & 1.000 & 0.294 & 0.120 & 0.147 & 0.146 & 0.833 & 0.842 & 0.833 & 0.397 & 0.079 & 0.786 & 1.000 & 0.998 \\
\hline$\chi^{2}$ & 0.063 & 1.000 & 0.157 & 0.081 & 0.098 & 0.087 & 0.549 & 0.572 & 0.555 & 0.098 & 0.045 & 0.562 & 0.990 & 0.977 \\
\hline $\begin{array}{l}\text { Power divergent family } \\
\quad(\lambda=2 / 3)\end{array}$ & 0.072 & 1.000 & 0.166 & 0.092 & 0.105 & 0.098 & 0.553 & 0.568 & 0.562 & 0.137 & 0.065 & 0.567 & 0.989 & 0.977 \\
\hline LRT & 0.026 & 1.000 & 0.210 & 0.080 & 0.094 & 0.089 & 0.763 & 0.788 & 0.778 & 0.000 & 0.001 & 0.753 & 0.999 & 0.998 \\
\hline Hellinger deviance & 0.044 & 1.000 & 0.221 & 0.111 & 0.114 & 0.128 & 0.738 & 0.762 & 0.729 & 0.001 & 0.004 & 0.779 & 0.998 & 0.992 \\
\hline Rayner & 0.062 & 1.000 & 0.256 & 0.127 & 0.114 & 0.141 & 0.724 & 0.761 & 0.757 & 0.121 & 0.027 & 0.656 & 0.999 & 0.996 \\
\hline Cramér-von Mises 1 & 0.051 & 1.000 & 0.140 & 0.075 & 0.073 & 0.074 & 0.534 & 0.525 & 0.517 & 0.294 & 0.077 & 0.550 & 0.985 & 0.974 \\
\hline Cramér-von Mises 2 & 0.052 & 1.000 & 0.206 & 0.097 & 0.097 & 0.094 & 0.715 & 0.703 & 0.693 & 0.343 & 0.078 & 0.703 & 0.998 & 0.995 \\
\hline Cramér-von Mises 3 & 0.053 & 1.000 & 0.184 & 0.091 & 0.090 & 0.088 & 0.678 & 0.663 & 0.651 & 0.339 & 0.080 & 0.673 & 0.997 & 0.992 \\
\hline Cramér-von Mises 4 & 0.052 & 1.000 & 0.152 & 0.077 & 0.078 & 0.077 & 0.550 & 0.540 & 0.532 & 0.265 & 0.069 & 0.551 & 0.986 & 0.977 \\
\hline Rescaled VT & 0.041 & 1.000 & 0.080 & 0.037 & 0.036 & 0.037 & 0.531 & 0.514 & 0.492 & 0.580 & 0.142 & 0.545 & 0.975 & 0.945 \\
\hline $\begin{array}{l}\text { Empirical integrated } \\
\text { distribution function }\end{array}$ & 0.055 & 1.000 & 0.244 & 0.108 & 0.108 & 0.104 & 0.770 & 0.759 & 0.751 & 0.438 & 0.096 & 0.737 & 0.996 & 0.993 \\
\hline $\begin{array}{l}\text { Efron double-exponential } \\
\text { family }\end{array}$ & 0.063 & 1.000 & 0.332 & 0.162 & 0.162 & 0.162 & 0.814 & 0.813 & 0.809 & 0.000 & 0.008 & 0.759 & 0.998 & 0.996 \\
\hline
\end{tabular}

$\dagger$ The Poisson parameter was set to $\lambda=5$. The sample size was $100(\alpha=5 \%, 10000$ replications $)$. 
Table 11. Calculated power for all the tests employed in the simulation study and for all the alternative distributions used $\dagger$

\begin{tabular}{|c|c|c|c|c|c|c|c|c|c|c|c|c|c|c|}
\hline \multirow[t]{2}{*}{ Test } & \multicolumn{14}{|c|}{ Powers for the following alternative distributions: } \\
\hline & (a) & (b) & (c) & (d) & (e) & $(f)$ & (g) & (h) & (i) & $(j)$ & ( $k$ ) & (l) & (m) & (n) \\
\hline 2nd cumulant & 0.063 & 1.000 & 0.881 & 0.449 & 0.464 & 0.448 & 1.000 & 1.000 & 1.000 & 0.999 & 0.466 & 1.000 & 1.000 & 1.000 \\
\hline VT & 0.068 & 1.000 & 0.888 & 0.460 & 0.477 & 0.461 & 1.000 & 1.000 & 1.000 & 0.999 & 0.447 & 1.000 & 1.000 & 1.000 \\
\hline Böhning & 0.069 & 1.000 & 0.888 & 0.463 & 0.480 & 0.464 & 1.000 & 1.000 & 1.000 & 0.999 & 0.456 & 1.000 & 1.000 & 1.000 \\
\hline Zelterman & 0.062 & 1.000 & 0.881 & 0.449 & 0.463 & 0.448 & 1.000 & 1.000 & 1.000 & 0.999 & 0.461 & 1.000 & 1.000 & 1.000 \\
\hline 3rd cumulant & 0.057 & 1.000 & 0.631 & 0.162 & 0.175 & 0.152 & 0.977 & 0.975 & 0.987 & 0.745 & 0.134 & 0.677 & 1.000 & 1.000 \\
\hline 4th cumulant & 0.057 & 0.829 & 0.272 & 0.106 & 0.097 & 0.096 & 0.565 & 0.602 & 0.693 & 0.001 & 0.017 & 0.378 & 0.971 & 0.996 \\
\hline Kocherlakota $(t=-0.05)$ & 0.051 & 1.000 & 0.112 & 0.079 & 0.095 & 0.075 & 0.581 & 0.545 & 0.506 & 0.066 & 0.051 & 0.665 & 0.999 & 0.960 \\
\hline Kocherlakota $(t=0.05)$ & 0.046 & 1.000 & 0.120 & 0.080 & 0.088 & 0.080 & 0.738 & 0.709 & 0.662 & 0.202 & 0.062 & 0.828 & 1.000 & 0.997 \\
\hline Kocherlakota $(t=0.125)$ & 0.048 & 1.000 & 0.157 & 0.106 & 0.104 & 0.102 & 0.866 & 0.853 & 0.791 & 0.423 & 0.090 & 0.916 & 1.000 & 1.000 \\
\hline Nass & 0.046 & 1.000 & 0.377 & 0.111 & 0.101 & 0.097 & 0.985 & 0.981 & 0.988 & 0.182 & 0.022 & 0.960 & 1.000 & 1.000 \\
\hline Gupta & 0.063 & 0.831 & 0.021 & 0.066 & 0.063 & 0.071 & 0.041 & 0.040 & 0.016 & 0.000 & 0.025 & 0.439 & 0.000 & 0.000 \\
\hline Baringhaus & 0.056 & 1.000 & 0.558 & 0.244 & 0.229 & 0.242 & 0.999 & 0.999 & 1.000 & 0.939 & 0.201 & 1.000 & 1.000 & 1.000 \\
\hline Nakamura & 0.050 & 1.000 & 0.695 & 0.229 & 0.219 & 0.237 & 1.000 & 1.000 & 1.000 & 0.961 & 0.183 & 1.000 & 1.000 & 1.000 \\
\hline Rueda & 0.053 & 1.000 & 0.522 & 0.236 & 0.222 & 0.230 & 0.999 & 0.999 & 0.999 & 0.919 & 0.196 & 1.000 & 0.996 & 1.000 \\
\hline $\mathrm{KS}$ & 0.053 & 1.000 & 0.470 & 0.164 & 0.150 & 0.169 & 0.992 & 0.997 & 0.984 & 0.881 & 0.158 & 0.997 & 1.000 & 1.000 \\
\hline McIntyre & 0.045 & 1.000 & 0.833 & 0.360 & 0.359 & 0.376 & 1.000 & 1.000 & 1.000 & 0.997 & 0.306 & 1.000 & 1.000 & 1.000 \\
\hline$\chi^{2}$ & 0.058 & 1.000 & 0.566 & 0.200 & 0.171 & 0.199 & 0.998 & 0.999 & 0.997 & 0.740 & 0.078 & 0.999 & 1.000 & 1.000 \\
\hline $\begin{array}{l}\text { Power divergent family } \\
\quad(\lambda=2 / 3)\end{array}$ & 0.054 & 1.000 & 0.527 & 0.174 & 0.149 & 0.165 & 0.997 & 0.999 & 0.995 & 0.776 & 0.089 & 0.999 & 1.000 & 1.000 \\
\hline LRT & 0.036 & 1.000 & 0.824 & 0.360 & 0.359 & 0.375 & 1.000 & 1.000 & 1.000 & 0.000 & 0.000 & 1.000 & 1.000 & 1.000 \\
\hline Hellinger deviance & 0.056 & 1.000 & 0.828 & 0.401 & 0.410 & 0.418 & 1.000 & 1.000 & 1.000 & 0.000 & 0.000 & 1.000 & 1.000 & 1.000 \\
\hline Rayner & 0.060 & 1.000 & 0.697 & 0.249 & 0.234 & 0.236 & 1.000 & 0.999 & 1.000 & 0.914 & 0.105 & 1.000 & 1.000 & 1.000 \\
\hline Cramér-von Mises 1 & 0.051 & 1.000 & 0.535 & 0.180 & 0.182 & 0.180 & 0.997 & 0.997 & 0.997 & 0.936 & 0.213 & 0.999 & 1.000 & 1.000 \\
\hline Cramér-von Mises 2 & 0.055 & 1.000 & 0.692 & 0.253 & 0.255 & 0.252 & 1.000 & 1.000 & 1.000 & 0.980 & 0.266 & 1.000 & 1.000 & 1.000 \\
\hline Cramér-von Mises 3 & 0.054 & 1.000 & 0.651 & 0.236 & 0.237 & 0.236 & 1.000 & 1.000 & 1.000 & 0.974 & 0.258 & 1.000 & 1.000 & 1.000 \\
\hline Cramér-von Mises 4 & 0.052 & 1.000 & 0.549 & 0.186 & 0.187 & 0.185 & 0.997 & 0.998 & 0.998 & 0.931 & 0.205 & 0.999 & 1.000 & 1.000 \\
\hline Rescaled VT & 0.047 & 1.000 & 0.726 & 0.256 & 0.257 & 0.255 & 1.000 & 1.000 & 1.000 & 0.998 & 0.434 & 1.000 & 1.000 & 1.000 \\
\hline $\begin{array}{l}\text { Empirical integrated } \\
\text { distribution function }\end{array}$ & 0.054 & 1.000 & 0.743 & 0.301 & 0.298 & 0.303 & 1.000 & 1.000 & 1.000 & 0.991 & 0.331 & 1.000 & 1.000 & 1.000 \\
\hline $\begin{array}{l}\text { Efron double-exponential } \\
\text { family }\end{array}$ & 0.073 & 1.000 & 0.825 & 0.404 & 0.405 & 0.399 & 1.000 & 1.000 & 1.000 & 0.000 & 0.000 & 1.000 & 1.000 & 1.000 \\
\hline
\end{tabular}

$\dagger$ The Poisson parameter was set to $\lambda=5$. The sample size was 500 ( $\alpha=5 \%, 10000$ replications). 
of the $\chi^{2}$-test, does not appear to perform well especially when the sample size is small.

So, it becomes obvious from the results that our choice of a test for testing the Poisson assumption against a variety of overdispersed and underdispersed alternatives should be one of the tests based on the variance-to-mean ratio. The LRT and the Hellinger deviance test perform well for the overdispersion case, but they require much computing. Moreover, their critical values are not known. The use of test statistic (8) would be preferable. The reason is that simulations showed that the distribution of the test statistic is close to the standard normal distribution.

\subsection{Alternatives with variance equal to the mean}

Tables 12-14 contain results concerning the equidispersed alternatives. A first glance reveals that in this case the tests based on the variance-to-mean ratio fail, as was expected. In addition, we can see that the power of all the tests is low for small sample sizes and small values of the mean of the

Table 12. Calculated power for all the tests employed in the simulation study and for the equidispersed alternative distributions used $\dagger$

\begin{tabular}{|c|c|c|c|c|c|c|c|c|c|}
\hline \multirow[t]{3}{*}{ Test } & \multicolumn{9}{|c|}{ Powers for the following alternative distributions and values of $n$ : } \\
\hline & \multicolumn{3}{|c|}{$n=50$} & \multicolumn{3}{|c|}{$n=100$} & \multicolumn{3}{|c|}{$n=500$} \\
\hline & (a) & (b) & (c) & (a) & (b) & (c) & (a) & (b) & (c) \\
\hline 2nd cumulant & 0.047 & 0.048 & 0.011 & 0.042 & 0.040 & 0.007 & 0.029 & 0.023 & 0.006 \\
\hline VT & 0.054 & 0.051 & 0.016 & 0.048 & 0.043 & 0.010 & 0.033 & 0.025 & 0.010 \\
\hline Böhning & 0.061 & 0.050 & 0.018 & 0.055 & 0.042 & 0.010 & 0.037 & 0.024 & 0.009 \\
\hline Zelterman & 0.047 & 0.047 & 0.009 & 0.041 & 0.039 & 0.008 & 0.028 & 0.023 & 0.006 \\
\hline 3rd cumulant & 0.044 & 0.041 & 0.025 & 0.033 & 0.035 & 0.057 & 0.020 & 0.084 & 0.677 \\
\hline 4 th cumulant & 0.048 & 0.047 & 0.337 & 0.045 & 0.073 & 0.715 & 0.079 & 0.518 & 1.000 \\
\hline Kocherlakota $(t=-0.05)$ & 0.052 & 0.050 & 0.086 & 0.049 & 0.061 & 0.162 & 0.054 & 0.167 & 0.671 \\
\hline Kocherlakota $(t=0.05)$ & 0.049 & 0.052 & 0.076 & 0.047 & 0.061 & 0.140 & 0.051 & 0.154 & 0.603 \\
\hline Kocherlakota $(t=0.125)$ & 0.050 & 0.051 & 0.069 & 0.050 & 0.059 & 0.124 & 0.052 & 0.139 & 0.536 \\
\hline Nass & 0.045 & 0.041 & 0.043 & 0.041 & 0.032 & 0.212 & 0.040 & 0.065 & 0.065 \\
\hline Gupta & 0.055 & 0.071 & 0.376 & 0.069 & 0.152 & 0.684 & 0.133 & 0.799 & 1.000 \\
\hline Baringhaus & 0.043 & 0.051 & 0.059 & 0.040 & 0.062 & 0.139 & 0.041 & 0.169 & 0.687 \\
\hline Nakamura & 0.045 & 0.049 & 0.169 & 0.042 & 0.064 & 0.442 & 0.049 & 0.311 & 1.000 \\
\hline Rueda & 0.051 & 0.049 & 0.046 & 0.048 & 0.055 & 0.103 & 0.043 & 0.121 & 0.470 \\
\hline $\mathrm{KS}$ & 0.056 & 0.055 & 0.095 & 0.054 & 0.068 & 0.218 & 0.060 & 0.209 & 0.857 \\
\hline McIntyre & 0.045 & 0.046 & 0.005 & 0.037 & 0.034 & 0.005 & 0.022 & 0.016 & 0.005 \\
\hline$\chi^{2}$ & 0.052 & 0.051 & 0.134 & 0.055 & 0.060 & 0.192 & 0.066 & 0.236 & 0.728 \\
\hline $\begin{array}{l}\text { Power divergent family } \\
\quad(\lambda=2 / 3)\end{array}$ & 0.049 & 0.063 & 0.123 & 0.051 & 0.074 & 0.228 & 0.063 & 0.271 & 0.756 \\
\hline LRT & 0.051 & 0.026 & 0.114 & 0.053 & 0.026 & 0.118 & 0.064 & 0.026 & 0.036 \\
\hline Hellinger deviance & 0.066 & 0.037 & 0.114 & 0.075 & 0.044 & 0.080 & 0.099 & 0.043 & 0.003 \\
\hline Rayner & 0.044 & 0.030 & 0.039 & 0.029 & 0.002 & 0.089 & 0.017 & 0.002 & 0.998 \\
\hline Cramér-von Mises 1 & 0.053 & 0.051 & 0.128 & 0.049 & 0.070 & 0.267 & 0.060 & 0.241 & 0.903 \\
\hline Cramér-von Mises 2 & 0.052 & 0.051 & 0.131 & 0.049 & 0.068 & 0.291 & 0.057 & 0.251 & 0.983 \\
\hline Cramér-von Mises 3 & 0.052 & 0.051 & 0.133 & 0.050 & 0.069 & 0.273 & 0.057 & 0.244 & 0.953 \\
\hline Cramér-von Mises 4 & 0.051 & 0.051 & 0.117 & 0.050 & 0.068 & 0.242 & 0.056 & 0.234 & 0.901 \\
\hline Rescaled VT & 0.042 & 0.048 & 0.031 & 0.038 & 0.046 & 0.028 & 0.029 & 0.042 & 0.040 \\
\hline $\begin{array}{l}\text { Empirical integrated } \\
\text { distribution function }\end{array}$ & 0.050 & 0.051 & 0.053 & 0.044 & 0.056 & 0.118 & 0.043 & 0.137 & 0.625 \\
\hline $\begin{array}{l}\text { Efron double-exponential } \\
\text { family }\end{array}$ & 0.046 & 0.048 & 0.013 & 0.041 & 0.042 & 0.009 & 0.028 & 0.029 & 0.010 \\
\hline
\end{tabular}

$\dagger$ The Poisson parameter was set to $\lambda=1(\alpha=5 \%, 10000$ replications). The three alternatives used had parameter vectors $(N, \alpha, b)$ equal to $(6,4,20),(4,2,6)$ and $(3,1,2)$. The smaller the value of $N$ is, the greater the difference from the Poisson distribution. 
Table 13. Calculated power for all the tests employed in the simulation study and for the equidispersed alternative distributions used $\dagger$

\begin{tabular}{|c|c|c|c|c|c|c|c|c|c|}
\hline \multirow[t]{3}{*}{ Test } & \multicolumn{9}{|c|}{ Powers for the following alternative distributions and values of $n$ : } \\
\hline & \multicolumn{3}{|c|}{$n=50$} & \multicolumn{3}{|c|}{$n=100$} & \multicolumn{3}{|c|}{$n=500$} \\
\hline & (a) & (b) & (c) & (a) & (b) & (c) & (a) & (b) & (c) \\
\hline 2nd cumulant & 0.047 & 0.045 & 0.034 & 0.044 & 0.042 & 0.036 & 0.030 & 0.033 & 0.024 \\
\hline VT & 0.056 & 0.047 & 0.047 & 0.053 & 0.044 & 0.043 & 0.037 & 0.036 & 0.026 \\
\hline Böhning & 0.056 & 0.048 & 0.047 & 0.053 & 0.045 & 0.043 & 0.037 & 0.037 & 0.027 \\
\hline Zelterman & 0.045 & 0.043 & 0.033 & 0.043 & 0.040 & 0.036 & 0.028 & 0.032 & 0.024 \\
\hline 3rd cumulant & 0.044 & 0.055 & 0.938 & 0.042 & 0.082 & 1.000 & 0.069 & 0.551 & 1.000 \\
\hline 4th cumulant & 0.047 & 0.071 & 0.518 & 0.046 & 0.129 & 0.811 & 0.092 & 0.840 & 1.000 \\
\hline Kocherlakota $(t=-0.05)$ & 0.052 & 0.063 & 0.577 & 0.052 & 0.083 & 0.830 & 0.079 & 0.311 & 1.000 \\
\hline Kocherlakota $(t=0.05)$ & 0.050 & 0.064 & 0.596 & 0.052 & 0.087 & 0.849 & 0.079 & 0.332 & 1.000 \\
\hline Kocherlakota $(t=0.125)$ & 0.053 & 0.062 & 0.602 & 0.056 & 0.084 & 0.851 & 0.084 & 0.332 & 1.000 \\
\hline Nass & 0.040 & 0.031 & 0.089 & 0.036 & 0.025 & 0.105 & 0.028 & 0.067 & 0.065 \\
\hline Gupta & 0.066 & 0.115 & 0.478 & 0.075 & 0.201 & 0.706 & 0.144 & 0.896 & 1.000 \\
\hline Baringhaus & 0.053 & 0.067 & 0.628 & 0.058 & 0.089 & 0.849 & 0.095 & 0.336 & 1.000 \\
\hline Nakamura & 0.044 & 0.059 & 0.968 & 0.044 & 0.084 & 1.000 & 0.069 & 0.536 & 1.000 \\
\hline Rueda & 0.053 & 0.064 & 0.567 & 0.057 & 0.084 & 0.801 & 0.089 & 0.311 & 1.000 \\
\hline $\mathrm{KS}$ & 0.050 & 0.054 & 0.823 & 0.051 & 0.068 & 0.994 & 0.063 & 0.287 & 1.000 \\
\hline McIntyre & 0.044 & 0.045 & 0.031 & 0.041 & 0.041 & 0.034 & 0.025 & 0.030 & 0.020 \\
\hline$\chi^{2}$ & 0.054 & 0.058 & 0.550 & 0.057 & 0.072 & 0.855 & 0.079 & 0.385 & 1.000 \\
\hline $\begin{array}{l}\text { Power divergent family } \\
\quad(\lambda=2 / 3)\end{array}$ & 0.051 & 0.056 & 0.527 & 0.052 & 0.069 & 0.842 & 0.070 & 0.384 & 1.000 \\
\hline LRT & 0.037 & 0.035 & 0.333 & 0.038 & 0.036 & 0.262 & 0.061 & 0.041 & 0.079 \\
\hline Hellinger deviance & 0.067 & 0.032 & 0.181 & 0.072 & 0.035 & 0.086 & 0.107 & 0.051 & 0.001 \\
\hline Rayner & 0.037 & 0.043 & 0.788 & 0.031 & 0.061 & 0.997 & 0.033 & 0.518 & 1.000 \\
\hline Cramér-von Mises 1 & 0.048 & 0.058 & 0.906 & 0.052 & 0.076 & 0.999 & 0.063 & 0.358 & 1.000 \\
\hline Cramér-von Mises 2 & 0.047 & 0.059 & 0.959 & 0.049 & 0.076 & 1.000 & 0.064 & 0.411 & 1.000 \\
\hline Cramér-von Mises 3 & 0.048 & 0.060 & 0.936 & 0.051 & 0.077 & 0.999 & 0.066 & 0.394 & 1.000 \\
\hline Cramér-von Mises 4 & 0.045 & 0.054 & 0.912 & 0.046 & 0.068 & 0.999 & 0.047 & 0.334 & 1.000 \\
\hline Rescaled VT & 0.042 & 0.050 & 0.026 & 0.039 & 0.047 & 0.035 & 0.031 & 0.049 & 0.038 \\
\hline $\begin{array}{l}\text { Empirical integrated } \\
\text { distribution function }\end{array}$ & 0.049 & 0.052 & 0.501 & 0.047 & 0.057 & 0.940 & 0.046 & 0.154 & 1.000 \\
\hline $\begin{array}{l}\text { Efron double-exponential } \\
\text { family }\end{array}$ & 0.042 & 0.036 & 0.000 & 0.036 & 0.029 & 0.000 & 0.017 & 0.006 & 0.000 \\
\hline
\end{tabular}

$\dagger$ The Poisson parameter was set to $\lambda=3(\alpha=5 \%, 10000$ replications). The three alternatives used had parameter vectors $(N, \alpha, b)$ equal to $(12,8,24),(8,4,6.67)$ and $(5,1,0.67)$. The smaller the value of $N$ is, the greater the difference from the Poisson distribution.

Poisson distribution. The latter can be explained by the small difference between the Poisson distribution and the alternative considered.

For smaller sample sizes, the test based on the fourth cumulant and the test proposed by Gupta seem to be preferable. Recall that the power of these tests was quite low when overdispersed and underdispersed alternatives were considered. Both tests lose their power with respect to other tests, for larger values of the mean, mainly because of the higher variability in the quantities used for calculating the statistic (high order sample moments).

As the sample size increases and the difference between the alternative and the corresponding Poisson distribution also increases, all the tests except those based on the variance-to-mean ratio behave well. The LRT and Hellinger deviance test are not meaningful in the equidispersed alternative cases.

The test proposed by Nakamura and Perez-Abreu (1993) seems to be preferable to the test 
Table 14. Calculated power for all the tests employed in the simulation study and for the equidispersed alternative distributions used ${ }^{\dagger}$

\begin{tabular}{|c|c|c|c|c|c|c|c|c|c|}
\hline \multirow[t]{3}{*}{ Test } & \multicolumn{9}{|c|}{ Powers for the following alternative distributions and values of $n$ : } \\
\hline & \multicolumn{3}{|c|}{$n=50$} & \multicolumn{3}{|c|}{$n=100$} & \multicolumn{3}{|c|}{$n=500$} \\
\hline & (a) & (b) & (c) & (a) & (b) & (c) & (a) & (b) & (c) \\
\hline 2nd cumulant & 0.045 & 0.044 & 0.060 & 0.044 & 0.042 & 0.065 & 0.037 & 0.034 & 0.065 \\
\hline VT & 0.057 & 0.047 & 0.081 & 0.056 & 0.046 & 0.077 & 0.049 & 0.036 & 0.066 \\
\hline Böhning & 0.057 & 0.048 & 0.081 & 0.057 & 0.046 & 0.076 & 0.049 & 0.037 & 0.066 \\
\hline Zelterman & 0.044 & 0.044 & 0.058 & 0.043 & 0.042 & 0.066 & 0.037 & 0.034 & 0.065 \\
\hline 3rd cumulant & 0.044 & 0.080 & 1.000 & 0.046 & 0.106 & 1.000 & 0.090 & 0.671 & 1.000 \\
\hline 4th cumulant & 0.045 & 0.076 & 0.214 & 0.046 & 0.103 & 0.296 & 0.077 & 0.639 & 0.778 \\
\hline Kocherlakota $(t=-0.05)$ & 0.061 & 0.069 & 0.737 & 0.064 & 0.075 & 0.926 & 0.101 & 0.197 & 1.000 \\
\hline Kocherlakota $(t=0.05)$ & 0.052 & 0.065 & 0.762 & 0.054 & 0.073 & 0.948 & 0.075 & 0.228 & 1.000 \\
\hline Kocherlakota $(t=0.125)$ & 0.052 & 0.070 & 0.772 & 0.051 & 0.078 & 0.961 & 0.077 & 0.270 & 1.000 \\
\hline Nass & 0.042 & 0.027 & 0.102 & 0.040 & 0.028 & 0.132 & 0.033 & 0.039 & 0.054 \\
\hline Gupta & 0.066 & 0.133 & 0.240 & 0.071 & 0.168 & 0.302 & 0.127 & 0.734 & 0.703 \\
\hline Baringhaus & 0.068 & 0.081 & 0.819 & 0.071 & 0.094 & 0.965 & 0.124 & 0.320 & 1.000 \\
\hline Nakamura & 0.047 & 0.068 & 1.000 & 0.047 & 0.081 & 1.000 & 0.068 & 0.480 & 1.000 \\
\hline Rueda & 0.061 & 0.076 & 0.801 & 0.064 & 0.089 & 0.955 & 0.114 & 0.310 & 1.000 \\
\hline KS & 0.052 & 0.066 & 0.999 & 0.051 & 0.072 & 1.000 & 0.066 & 0.282 & 1.000 \\
\hline McIntyre & 0.044 & 0.044 & 0.069 & 0.043 & 0.042 & 0.086 & 0.035 & 0.031 & 0.071 \\
\hline$\chi^{2}$ & 0.052 & 0.063 & 0.578 & 0.051 & 0.069 & 0.965 & 0.069 & 0.314 & 1.000 \\
\hline $\begin{array}{l}\text { Power divergent family } \\
\quad(\lambda=2 / 3)\end{array}$ & 0.054 & 0.059 & 0.592 & 0.053 & 0.065 & 0.968 & 0.068 & 0.303 & 1.000 \\
\hline LRT & 0.038 & 0.029 & 0.433 & 0.039 & 0.030 & 0.417 & 0.063 & 0.032 & 0.379 \\
\hline Hellinger deviance & 0.044 & 0.045 & 0.165 & 0.045 & 0.043 & 0.088 & 0.075 & 0.043 & 0.002 \\
\hline Rayner & 0.041 & 0.048 & 0.990 & 0.039 & 0.065 & 1.000 & 0.057 & 0.446 & 1.000 \\
\hline Cramér-von Mises 1 & 0.050 & 0.064 & 1.000 & 0.051 & 0.073 & 1.000 & 0.066 & 0.336 & 1.000 \\
\hline Cramér-von Mises 2 & 0.051 & 0.064 & 1.000 & 0.052 & 0.074 & 1.000 & 0.069 & 0.371 & 1.000 \\
\hline Cramér-von Mises 3 & 0.051 & 0.065 & 1.000 & 0.052 & 0.075 & 1.000 & 0.070 & 0.359 & 1.000 \\
\hline Cramér-von Mises 4 & 0.046 & 0.059 & 1.000 & 0.046 & 0.066 & 1.000 & 0.049 & 0.306 & 1.000 \\
\hline Rescaled VT & 0.041 & 0.051 & 0.027 & 0.038 & 0.048 & 0.043 & 0.030 & 0.047 & 0.046 \\
\hline $\begin{array}{l}\text { Empirical integrated } \\
\text { distribution function }\end{array}$ & 0.050 & 0.053 & 0.943 & 0.048 & 0.056 & 1.000 & 0.053 & 0.138 & 1.000 \\
\hline $\begin{array}{l}\text { Efron double-exponential } \\
\text { family }\end{array}$ & 0.047 & 0.051 & 0.045 & 0.047 & 0.051 & 0.045 & 0.044 & 0.047 & 0.049 \\
\hline
\end{tabular}

$\dagger$ The Poisson parameter was set to $\lambda=5(\alpha=5 \%, 10000$ replications). The three alternatives used had parameter vectors $(N, \alpha, b)$ equal to $(18,12,31.2),(12,6,8.4)$ and $(7,1,0.4)$. The smaller the value of $N$ is, the greater the difference from the Poisson distribution.

based on the EGF, whereas the Cramér-von Mises tests are preferable to the Kolmogorov-type tests. From the results, we can conclude that an indisputable choice that performs better in every case does not exist.

\section{Conclusions}

In this paper, several tests proposed in the literature for testing whether data come from the Poisson distribution have been compared. The results obtained can be quite useful in practice, especially in guiding one's choice of the appropriate test, as, for the first time, the performance of all those tests has been examined. In the past, only comparisons between very few tests have been made, mainly to demonstrate the advantage of a new test over an existing test (see, for example, the comparisons in Rueda et al. (1991), Nakamura and Perez-Abreu (1993), Kim and Park (1992) and Henze and Klar (1995)). 
When the alternatives show overdispersion with an index larger than 1.25, all the tests had a satisfactory performance. For overdispersed and underdispersed alternatives, the test proposed by Böhning (1994) seems to be preferable. In general, all the tests that are based on the sample variance to the sample mean ratio have similar power levels. The advantage, however, of this test is that it is easily computed and the distribution of its test statistic can be very satisfactorily approximated by a standard normal distribution. In contrast, all the tests based on the variance-tomean ratio fail when the alternative is equidispersed.

Tests based on the PGF show a more balanced behaviour against every kind of alternative. Differences between them are rather small.

\section{Acknowledgements}

The authors would like to thank the referees for their constructive comments.

\section{References}

Albrecht, P. (1980) On the correct use of the chi-square goodness of fit test. Scand. Act. J., 7, 149-160.

Anderson, J. and Siddiqui, M. (1994) The sampling distribution of the index of dispersion. Communs Statist. Theory Meth., 23, 897-911.

Baglivo, J., Olivier, D. and Pagano, M. (1992) Methods for exact goodness of fit tests. J. Am. Statist. Ass., 87, $464-469$.

Baringhaus, L. and Henze, N. (1992) A goodness of fit test for the Poisson distribution based on the empirical generating function. Statist. Probab. Lett., 13, 269-274.

Bartko, J. J., Greenhouse, S. W. and Patlak, C. S. (1968) On expectations of some functions of Poisson variates. Biometrics, 24, 97-102.

Bartoo, J. and Puri, P. S. (1967) On optimal asymptotic tests of composite statistical hypotheses. Ann. Math. Statist., 38, $1845-1852$.

Basu, A. and Sarkar, S. (1994) On disparity based goodness-of-fit tests for multinomial models. Statist. Probab. Lett., 19, $307-312$.

Bateman, G. (1950) The power of the $\mathrm{X}^{2}$ index of dispersion when Neyman's contagious distribution is the alternate hypothesis. Biometrika, 37, 59-63.

Best, D. J. and Rayner, J. C. W. (1999) Goodness of fit for the Poisson distribution. Statist. Probab. Lett., 44, $259-265$.

Bhalerao, N., Gurland, J. and Tripathi, R. (1980) A method of increasing power of a test for the negative binomial and Neyman type A distributions. J. Am. Statist. Ass., 75, 934-938.

Böhning, D. (1994) A note on a test for Poisson overdispersion. Biometrika, 81, 418-419.

Böhning, D., Dietz, E., Schaub, R., Schlattmann, P. and Lindsay, B. (1994) The distribution of the likelihood ratio for mixtures of densities from the one-parameter exponential family. Ann. Inst. Statist. Math., 46, 373-388.

Campbell, D. B. and Oprian, C. A. (1979) On the Kolmogorov-Smirnov test for the Poisson distribution with unknown mean. Biometr. J., 21, 17-24.

Cochran, W. (1954) Some methods of strengthening the $\mathrm{X}^{2}$ goodness of fit test. Biometrics, 10, 417-451.

Collings, B. and Margolin, B. H. (1985) Testing goodness of fit for the Poisson assumption when observations are not identically distributed. J. Am. Statist. Ass., 80, 411-418.

Conover, W. J. (1972) A Kolmogorov goodness of fit test for discontinuous distributions. J. Am. Statist. Ass., 67, 591-600.

Consul, P. C. (1989) Generalized Poisson Distributions: Properties and Applications. New York: Dekker.

Cox, D. R. (1983) Some remarks on overdispersion. Biometrika, 70, 269-274.

Dahiya, R. C. and Gurland, J. (1969) Functions of the sample mean and sample variance of a Poisson variate. Biometrics, 25, $171-173$.

Darwin, R. (1957) The power of the Poisson index of dispersion. Biometrika, 44, 286-289.

Devroye, L. (1992) Non-uniform Random Variate Generation. New York: Springer.

Efron, B. (1986) Double exponential families and their use in generalized linear regression. J. Am. Statist. Ass., 81, $709-721$.

Efron, B. and Tibshirani, T. (1993) The Jackknife and the Bootstrap. New York: Dekker.

Epps, T. W. (1995) A test of fit for lattice distributions. Communs Statist. Theory Meth., 24, 1455-1479.

Gart, J. and Pettigrew, H. (1970) On the conditional moments of the K-statistics for the Poisson distribution. Biometrika, 57, 661-664.

Gelfand, A. and Dalal, S. (1990) A note on overdispersed exponential families. Biometrika, 77, 55-64.

Gupta, A. K., Mori, T. F. and Szekely, G. Z. (1994) Testing for Poissonity-normality vs other infinite divisibility. Statist. Probab. Lett., 19, 245-248.

Henze, N. (1996) Empirical distribution function goodness of fit tests for discrete models. Can. J. Statist., 24, 81-93. 
Henze, N. and Klar, B. (1995) Bootstrap based goodness of fit tests for the generalized Poisson model. Communs Statist. Theory Meth., 24, 1875-1896.

(1996) Properly rescaled components of smooth tests of fit are diagnostic. Aust. J. Statist., 38, 61-74.

Hinz, P. and Gurland, J. (1970) A test of fit for the negative binomial and other contagious distributions. J. Am. Statist. Ass., 65, 887-903.

Hoaglin, D. C. (1980) A Poissoness plot. Am. Statistn, 34, 146-149.

Hoel, P. G. (1943) On indices of dispersion. Ann. Math. Statist., 14, 155-162.

Horn, S. D. (1977) Goodness of fit tests for discrete data: a review and an application to a health impairment scale. Biometrics, 33, 237-248.

Johnson, N. L., Kotz, S. and Kemp, A. W. (1992) Univariate Discrete Distributions, 2nd edn. New York: Wiley.

Karlis, D. and Xekalaki, E. (1998a) Robust inference for finite Poisson mixtures. Technical Report 49. Department of Statistics, Athens University of Economics and Business, Athens. $81-103$.

(1999) On testing for the number of components in finite Poisson mixture models. Ann. Inst. Statist. Math., 51, $149-162$.

Kharshikar, A. V. (1970) On the expected value of $S^{2} / X$. Biometrics, 26, 343-346.

Kim, B. S. (1988) Some remarks on locally most powerful unbiased tests for the detection of mixtures of Poisson and binomial distributions. Communs Statist. Theory Meth., 17, 3733-3741.

Kim, B. S. and Park, C. (1992) Some remarks on testing the goodness-of-fit for the Poisson assumption. Communs Statist. Theory Meth., 21, 979-995.

Klar, B. (1999) Goodness of fit tests for discrete models based on the integrated distribution function. Metrika, 49, 53-69.

Kocherlakota, S. and Kocherlakota, K. (1986) Goodness of fit for discrete distributions. Communs Statist. Theory Meth., 15, 815-829.

Kyriakoussis, A., Li, G. and Papadopoulos, A. (1998) On characterization and goodness of fit test of some discrete distribution families. J. Statist. Planng Inf., 74, 215-228.

Lee, S. (1998) Detecting departures from a Poisson model. Communs Statist. Theory Meth., 25, 1201-1210.

Lindsay, B. (1986) Exponential family mixtures models with least square estimators. Ann. Statist., 14, $124-137$.

Lindsay, B. and Roeder, K. (1992) Residuals diagnostics for mixture models. J. Am. Statist. Ass., 87, 785-794.

McLachlan, G. J. (1987) On bootstrapping the likelihood ratio test statistic for the number of components in a normal mixture. Appl. Statist., 36, 318-324.

Moran, P. (1973) Asymptotic properties of homogeneity tests. Biometrika, 60, 69-85.

Nakamura, M. and Perez-Abreu, V. (1993) Use of an empirical probability generating function for testing a Poisson model. Can. J. Statist., 21, 149-156.

Nass, C. (1959) The $\mathrm{X}^{2}$ test for small expectations in contingency tables with special reference to accidents and absenteism. Biometrika, 46, 365-385.

Ord, J. K. (1967) Graphical methods for a class of discrete distributions. J. R. Statist. Soc. A, 130, 232-238.

Pahl, N. (1969) On testing for goodness of fit of the negative binomial distribution when expectations are small. Biometrics, 25, $142-151$.

Perry, J. N. and Mead, R. (1979) On the power of the index of dispersion test to detect spatial pattern. Biometrics, 35, 613-622.

Pettigrew, H. and Mohler, W. (1967) A rapid test for the Poisson distribution using the range. Biometrics, 23, $685-692$.

Potthoff, R. E. and Whittinghill, M. (1966) Testing for homogeneity: II, The Poisson distribution. Biometrika, 53, $183-190$.

Rayner, J. C. W. and Best, D. J. (1988) Smooth goodness of fit tests for regular distributions. Communs Statist. Theory Meth., 17, 3235-3267.

Rayner, J. C. W. and McIntyre, R. (1985) Use of the score statistic for testing goodness of fit of some generalised distributions. Biometr. J., 27, 159-165.

Read, T. and Cressie, N. (1988) Goodness of Fit Statistics for Discrete Multivariate Data. New York: Springer.

Rueda, R. and O'Reilly, F. (1999) Tests of fit for discrete distributions based on the probability generating function. Communs Statist. Simuln Computn, 28, 259-274.

Rueda, R., Perez-Abreu, V. and O'Reilly, F. (1991) Goodness of fit for the Poisson distribution based on the probability generating function. Communs Statist. Theory Meth., 20, 3093-3110.

Santner, T. and Duffy, D. (1989) The Statistical Analysis of Discrete Data. New York: Springer.

Selby, B. (1965) The index of dispersion as a test statistic. Biometrika, 52, 627-629.

Sichel, H. S. (1975) On a distribution law for word frequencies. J. Am. Statist. Ass., 70, 542-547.

Spinelli, J. J. and Stephens, M. A. (1997) Cramer Von-Mises tests of fit for the Poisson distribution. Can. J. Statist., 25, 257-268.

Stute, W., Manteiga, W. G. and Quindmil, M. P. (1993) Bootstrap based goodness of fit tests. Metrika, 40, $243-256$.

Titterington, D. M., Smith, A. F. M. and Makov, U. E. (1985) Statistical Analysis of Finite Mixtures Distributions. New York: Wiley.

Wood, C. and Altavela, M. (1978) Large sample results for the Kolmogorov-Smirnov statistics for discrete distributions. Biometrika, 65, 235-239.

Zelterman, D. (1988) Homogeneity tests against central-mixture alternatives. J. Am. Statist. Ass., 83, $179-182$. 